\title{
A Note on the New Glimm Functional for General Systems of Hyperbolic Conservation Laws
}

\author{
Jiale Hua and Tong Yang \\ Department of Mathematics \\ City University of Hong Kong \\ Kowloon, Hong Kong
}

\begin{abstract}
For systems of hyperbolic conservation laws, a new Glimm functional was recently constructed when the linearly degenerate manifold in each characteristic field is either the whole space or it consists of a finitely many smooth and transversal manifolds of co-dimension one. This new functional leads to the neat consistency and convergence rate estimation of the Glimm scheme. In this paper, by the motivation of the result in [2, we show that the corresponding new Glimm functional can be constructed for general systems only under the strict hyperbolicity assumption.
\end{abstract}

\section{Introduction}

Consider the Cauchy problem for a one dimensional system of conservation laws

$$
\begin{cases}u_{t}+f_{x}(u)=0, & t \geq 0,-\infty<x<\infty \\ u(x, 0)=u_{0}(x), & -\infty<x<\infty\end{cases}
$$

where $u \in \mathbb{R}^{n}, u \rightarrow f(u)$ is a smooth vector valued map defined in an open set $\Omega \subset \mathbb{R}^{n}$.

As usual, the system in 1.1 is assumed to be strictly hyperbolic, that is, for every $u \in \Omega$ the matrix $A(u)=\nabla_{u} f(u)$ has $\mathrm{n}$ real distinct eigenvalues

$$
\lambda_{1}(u)<\lambda_{2}(u)<\ldots<\lambda_{n}(u) .
$$


And correspondingly, there are $n$ linearly independent right eigenvectors denoted by

$$
r_{1}(u), r_{2}(u), \ldots, r_{n}(u) .
$$

One of the features of the hyperbolic systems is the formation of the shock waves. For this, there have been extensive studies on the well-posedness theory and the solution behavior, etc, cf. [4, 5, 7, 9, 10, 11, 12, 13, 17, 18, 23, 24] and the references therein.

In particular, the vanishing viscosity limit of the solutions to the hyperbolic system with artificial viscosity was established in [4]. That is, the solutions $u^{\epsilon}$ to the system

$$
\begin{cases}u_{t}^{\epsilon}+A\left(u^{\epsilon}\right) u_{x}^{\epsilon}=\epsilon u_{x x}^{\epsilon}, & t \geq 0,-\infty<x<\infty, \\ u^{\epsilon}(x, 0)=u_{0}(x), & -\infty<x<\infty\end{cases}
$$

converges to a unique solution of the hyperbolic Cauchy problem 1.1 under the assumption of small total variation.

For later presentation, we firstly recall the following basic definition of the characteristic fields, cf. [18].

Definition 1.1. For $i \in\{1,2, \ldots, n\}$, the $i$-th characteristic field is called genuinely nonlinear in $\Omega$ if

$$
\nabla \lambda_{i} \cdot r_{i} \neq 0, \quad \text { for all } u \in \Omega
$$

while the $i$-th characteristic field is called linearly degenerate if

$$
\nabla \lambda_{i} \cdot r_{i}=0, \quad \text { for all } u \in \Omega
$$

In the case when each characteristic field is either genuinely nonlinear or linearly degenerate, the global existence was established in the fundamental work of Glimm 12] in the framework of solutions with small total variation. This is achieved by the introduction of the Glimm scheme and the use of the Riemann problems as building blocks. Moreover, a deterministic version of the Glimm scheme was later given in 19 .

The Glimm functional introduced in 12 is a key component in the subsequent research in this direction. The functional measures the interaction potential of waves which is used to control the total variation of the solution. The 
decrease of the functional also plays an important role in the wave front tracking algorithm.

Use $\left\{S_{t} ; t>0\right\}$ to denote the standard Riemann semigroup generated by (1.1) whose trajectory $S_{t} u_{0}$ is a solution to the Cauchy problem. The $\mathrm{L}^{1}$ stability theory, cf. 4, 6, 7, 16, 21, guarantees the existence of such a semigroup. From the uniqueness of the solution to problem (1.1), the approximate solutions constructed by the deterministic version of the Glimm scheme converge to $S_{t} u_{0}$ as the mesh size tends to zero. The convergence rate was proved to be $o(1) s^{\frac{1}{2}}|\ln s|$ when each characteristic field is either genuinely nonlinear or linearly degenerate, where $s$ is the mesh size, 8 .

For general systems, the solution to the Riemann problem has different structure so that the Cauchy problem exhibits richer nonlinear phenomena. To estimate the wave interactions, one uses the same Glimm functional for waves in different families but a different one for waves in the same family.

More precisely, in $14,20,22$, the systems under the following assumption are studied:

For each characteristic field, the linear degenerate manifold $L D_{i} \equiv\left\{u: \nabla \lambda_{i}(u) \cdot r_{i}(u)=0\right\}$ is either the whole space or it

$(H)$ consists of a finite number of smooth manifolds of codimension one, which are transversal to the characteristic vector $r_{i}(u)$.

In fact, a "cubic" functional was introduced in 20 and was used in 22 in order to take care of the wave interactions globally. The functional used in 2022 is defined by the product of the strengths of two interacting waves and their effective "interaction" angle. Based on this improvement to the classical Glimm functional, the existence theory with the wave tracing argument for general systems under the assumption $(\mathrm{H})$ was established in $[22$. However, this functional is not satisfactory in proving the consistency and the convergence rate of the Glimm scheme. In fact, the consistency of the Glimm scheme was proved in 22 by carefully and artificially dividing the waves into groups according to their wave strengths in comparison with the grid size to some power. And the convergence rate of the Glimm scheme was shown to be $o(1) s^{\frac{1}{4}}|\ln s|$ in $[25$ and then $o(1) s^{\frac{1}{3}}|\ln s|$ in 15 , which are slower than the one given in 8 under the condition that each characteristic field is genuinely nonlinear or linearly degenerate. 
Recently, in [14], a new Glimm functional for wave interactions in the same family is constructed so that the Glimm theory can now be presented in an elegant way under the assumption $(\mathrm{H})$. In fact, the new Glimm functional for the wave interactions in the same family is optimal in the following sense. First, it yields a clear and complete proof of the consistency of the Glimm scheme. Then it leads to the proof of the same order of convergence rate for the general systems under the condition $(\mathrm{H})$ as for those under the condition that each characteristic field is genuinely nonlinear or linearly degenerate. Finally, it has the same decay effect as the classical one introduced by Glimm when the assumption of genuine nonlinearity is imposed. Accordingly, the Glimm scheme for general systems under the assumption $(\mathrm{H})$ can be analyzed satisfactorily without any artificial adjustment.

The motivation of this paper is the study on very general hyperbolic systems given in 2, 3, 4, In particular, a Glimm functional is constructed in [2,3] for the systems without the assumption $(\mathrm{H})$, which can be viewed as an elegant generalization of the one in 20,22 in the integral form except that every wave in the same family is considered as approaching.

With the integral form of Glimm functional for systems without the assumption $(\mathrm{H})$ given in $[2]$, we can now combine it with the new Glimm functional introduced in 14 to define a Glimm functional for systems without the assumption $(\mathrm{H})$ so that all the properties induced by the new Glimm functional under the assumption $(\mathrm{H})$ can still hold without this assumption.

Precisely, the following Glimm type functional will be defined.

$$
F(J) \equiv L(J)+M Q(J),
$$

where

$$
\begin{aligned}
L(J)= & \sum\{|\alpha|: \alpha \text { any wave crossing } \mathrm{J}\}, \quad Q(J)=Q_{d}(J)+\frac{1}{4} Q_{s}(J), \\
Q_{d}(J)= & \sum\{|\alpha||\beta|: \text { interacting waves } \alpha \text { and } \beta \text { of distinct } \\
& \quad \text { characteristic fields crossing } \mathrm{J}\}, \\
Q_{s}(J)= & \sum_{i=1}^{n} Q_{s}^{i} .
\end{aligned}
$$

Here $|\alpha|$ is the wave strength of a wave $\alpha, M>0$ is a sufficiently large constant, $J$ is any space-like curve. An $i$-wave $\alpha_{i}$, that is, a wave in the $i$-th family, on the left and a $j$-wave $\beta_{j}$ on the right are said to be approaching, if $i>j$. 




Figure 1: Shock splitting

The definition of $Q_{s}$ is complicated because it is closely related to the construction of $\mathrm{i}$-wave curve in the general system, cf. 22. Thus, its definition is postponed to the next section. Intuitively, its form can be illustrated by considering two $i$-th family shocks $\alpha, \beta$ ( $\alpha, \beta$ of the same sign) with speeds $\sigma_{\alpha}, \sigma_{\beta}$ respectively. In this case, the interaction functional $Q_{s}$ corresponding to these two waves is just

$$
Q_{s}(\alpha, \beta)=\frac{\int_{0}^{|\alpha|} \int_{0}^{|\beta|}\left|\sigma_{\alpha}(\xi)-\sigma_{\beta}\left(\xi^{\prime}\right)\right| \mathrm{d} \xi \mathrm{d} \xi^{\prime}}{t . v \cdot(\alpha, \beta)_{i}}
$$

where t.v. $(\alpha, \beta)_{i}$ is the sum of all i-waves lying between $\alpha$ and $\beta$, including $\alpha$ and $\beta$, and the integral is along the re-defined $i$-wave curves for $\alpha$ and $\beta$ introduced by 2 which will be recalled in the next section.

However, the functional $Q_{s}=\sum_{\alpha, \beta} Q_{s}(\alpha, \beta)$ with $\alpha, \beta$ being in the same family may not be decreasing through wave interactions. Its value may increase due to the shock splitting as shown in the following simple example, cf. Figure 1. That is, the denominator t.v. $\left(\alpha_{2}, \gamma\right)_{i}$ after shock splitting: $\alpha \rightarrow \alpha_{1}+\alpha_{2}$ is smaller than the one before the shock splitting: t.v. $(\alpha, \gamma){ }_{i}$. Hence there is an increase in the functional, and such increase can not be controlled by the cancellation or decrease in the Glimm functional. One of the key observations in this paper is that the increase in $Q_{s}$ due to shock splitting can be compensated by shock merging and eventually the magnitude of oscillation of this kind in $Q_{s}$ will be shown to be bounded. And this is essential for the use of the new Glimm functional in the proof of consistency and convergence rate of the Glimm scheme.

With the above preparation, the main theorem on the new Glimm functional can be stated as follows. 


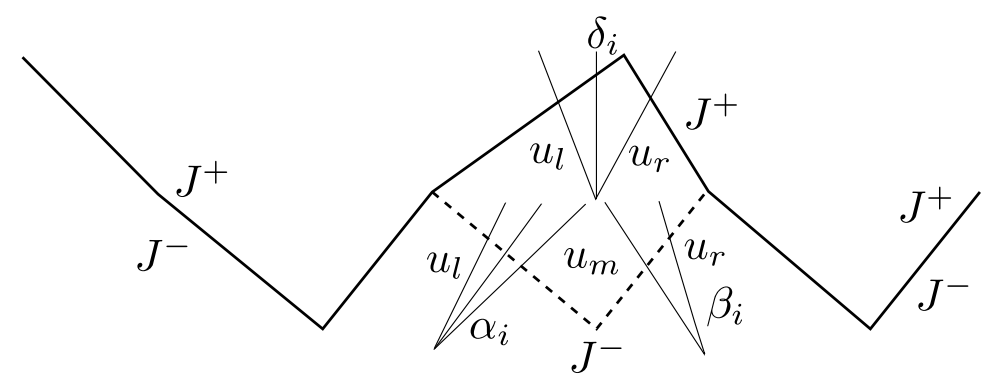

Figure 2: The wave interaction

Theorem 1.1. (i) Suppose that there is no shock splitting in the wave interaction. Let $u_{l}, u_{m}$ and $u_{r}$ be three nearby states and the Riemann problem $\left(u_{l}, u_{m}\right)$ and $\left(u_{m}, u_{r}\right)$ and $\left(u_{l}, u_{r}\right)$ be solved by waves $\alpha_{1}, \cdots, \alpha_{n}, \beta_{1}, \cdots, \beta_{n}$ and $\delta_{1}, \cdots, \delta_{n}$ respectively, see Figure $2 J^{+}$and $J^{-}$are two mesh curves. Denote the interaction potential before and after the interaction by $Q^{-}$and $Q^{+}$and their difference $Q^{+}-Q^{-}$by $\Delta Q$. Similar definitions hold for $L, F$.

Then

$$
\Delta F=\Delta L+M \Delta Q \leq-c\{Q(\alpha, \beta)+C(\alpha, \beta)\},
$$

where $C(\alpha, \beta)$ is the total cancellation in the interaction and $Q(\alpha, \beta)$ is the amount of interaction potential of $\alpha$ and $\beta$ which will be given precisely in the next section. Here, $c$ is some positive constant depending only on the system.

(ii) On the other hand, if there exists shock splitting, F may increase. However, for any given time $T>0$, we have

$$
F(T)-F(0) \leq O(1)\left(\text { Tot. Var. } u_{0}\right)^{2} .
$$

Remark 1.1. Note that the new Glimm functional may not be decreasing in time because of the definition of $Q_{s}(t)$ given later. However, the new Glimm functional consists of two parts, $G(t)$ and $S(t)$ as shown later in the proof. $G$ is non-increasing like the classical Glimm functional and the decrease of $G$ can be used to control the product of wave strength and variation of its propagation speed. On the other hand, $S(t)$ can be either positive or negative which represents the oscillation in the interaction potential due to the shock splitting. And it will be shown later that the absolute value of $S(t)$ is uniformly bounded by the total variation of the initial data to the second power. 
As an application of this Glimm functional, one obtain the optimal convergence rate of Glimm scheme o(1) $\sqrt{s}|\ln (s)|$ for general hyperbolic systems as in 8 for systems under the assumption (H). Here $s$ is the grid size of the Glimm scheme. Note that another work on this problem can be found in [1].

The rest of the paper will be arranged as follows. In the next section, we will introduce the new Glimm functional and prove the interaction estimates. The application of the new functional to the convergence rate will be given in the last section.

\section{Glimm Functional and Interaction Estimates}

To study the Cauchy problem (1.1), it is important to understand the Riemann problem first, in which the initial data has the following simple form:

$$
u_{0}(x)= \begin{cases}u^{-}, & \text {for } \quad x<0 \\ u^{+}, & \text {for } \quad x>0\end{cases}
$$

To solve the Riemann problem, let us recall the approach introduced in 3.4 . Consider a travelling wave for the viscous hyperbolic system

$$
u_{t}+A(u) u_{x}=u_{x x}
$$

with propagation speed $\sigma$. The equation can be written as a first order system on $\mathbb{R}^{n} \times \mathbb{R}^{n} \times \mathbb{R}$ :

$$
\left\{\begin{array}{l}
\dot{u}=v \\
\dot{v}=(A(u)-\sigma) v, \\
\dot{\sigma}=0 .
\end{array}\right.
$$

The center subspace $\mathcal{N}$ for the system linearized at $\left(u^{0}, 0, \lambda_{i}^{0}\right)$ consists of all vectors $(u, v, \sigma) \in \mathbb{R}^{n} \times \mathbb{R}^{n} \times \mathbb{R}$ such that

$$
V_{j}=0, \text { for all } j \neq i,
$$

where $V_{j}=\left\langle l_{j}^{0}, v\right\rangle$. Here $l_{j}^{0}, r_{j}^{0}$, are the left and right eigenvectors of $A\left(u^{0}\right)$ corresponding to the eigenvalue $\lambda_{i}^{0} \equiv \lambda_{i}\left(u^{0}\right)$. And these eigenvectors are normalized as follows:

$$
l_{i}^{0} r_{j}^{0}=\delta_{i j}, \quad\left|r_{j}^{0}\right|=1, \text { for all } i, j=1, \cdots, n \text {. }
$$


For each $i=1, \cdots, n$, by the Center Manifold Theorem, there exists a smooth manifold $\mathcal{M}_{i} \subset \mathbb{R}^{n+n+1}$, tangent to $\mathcal{N}$ at $\left(u^{0}, 0, \lambda_{i}^{0}\right)$, which is locally invariant under the flow 2.2 . This manifold can be written in the following form

$$
\begin{gathered}
\mathcal{M}_{i}=\left\{\left(u, v, \sigma_{i}\right) ; v=v_{i} \tilde{r}_{i}\left(u, v_{i}, \sigma_{i}\right)\right\}, \text { with } v_{i}=\left\langle l_{i}^{0}, v\right\rangle, \\
\left\langle l_{j}^{0}, \tilde{r}_{i}\left(u, v_{i}, \sigma_{i}\right)\right\rangle= \begin{cases}1, & i=j, \\
0, & i \neq j .\end{cases}
\end{gathered}
$$

Given the manifold $\mathcal{M}_{i}$, we can define the generalized eigenvalue $\tilde{\lambda}_{i}\left(u, v_{i}, \sigma_{i}\right)=$ $\left\langle l_{i}^{0}, A(u) \tilde{r}_{i}\left(u, v_{i}, \sigma_{i}\right)\right\rangle$ corresponding to the generalized eigenvector $\tilde{r}_{i}\left(u, v_{i}, \sigma_{i}\right)$.

The two smooth functions $\tilde{r}_{i}, \tilde{\lambda}_{i}$ defined on the $n+2$ variables $\left(u, v_{i}, \sigma_{i}\right) \in$ $\mathbb{R}^{n} \times \mathbb{R} \times \mathbb{R}$ have the following properties

$$
\tilde{r}_{i}\left(u^{0}, 0, \lambda_{i}^{0}\right)=r_{i}^{0}, \quad \tilde{\lambda}_{i}\left(u^{0}, 0, \lambda_{i}^{0}\right)=\lambda_{i}^{0}, \quad \frac{\partial}{\partial \sigma_{i}} \tilde{\lambda}_{i}\left(u^{0}, 0, \lambda_{i}^{0}\right)=0 .
$$

And for some constant $C_{0}>0$, it holds

$$
\left|\frac{\partial}{\partial v_{i}} \tilde{\lambda}_{i}\left(u, v_{i}, \sigma_{i}\right)\right| \leq C_{0}\left|u-u_{0}\right|, \quad\left|\frac{\partial}{\partial \sigma_{i}} \tilde{\lambda}_{i}\left(u, v_{i}, \sigma_{i}\right)\right| \leq C_{0}\left|v_{i}\right|\left|u-u_{0}\right| .
$$

Given the functions $\tilde{r}_{i}, \tilde{\lambda}_{i}$ and any fixed $s$ and $\bar{u}$ with $\left|\bar{u}-u^{0}\right|+|s|$ being sufficiently small, a curve $T_{s}^{i}[\bar{u}](i=1, \cdots, n)$ can be constructed by solving for $0 \leq \tau \leq s$ the integral system

$$
\left\{\begin{array}{l}
u(\tau)=\bar{u}+\int_{0}^{\tau} \tilde{r}_{i}\left(u(\xi), v_{i}(\xi), \sigma_{i}(\xi)\right) \mathrm{d} \xi \\
v_{i}(\tau)=\tilde{f}_{i}(\tau ; \gamma)-\operatorname{conv}_{[0, s]} \tilde{f}_{i}(\tau ; \gamma), \\
\sigma_{i}(\tau)=\frac{d}{d \tau} \operatorname{conv}_{[0, s]} \tilde{f}_{i}(\tau ; \gamma),
\end{array}\right.
$$

where $\tilde{f}_{i}$ is the scalar reduced flux function

$$
\begin{gathered}
\tilde{f}_{i}(\tau ; \gamma) \equiv \int_{0}^{\tau} \tilde{\lambda}_{i}\left(u(\xi), v_{i}(\xi), \sigma_{i}(\xi)\right) \mathrm{d} \xi, \quad \gamma(\tau)=\left(u(\tau), v_{i}(\tau), \sigma_{i}(\tau)\right), \\
\operatorname{conv}_{[a, b]} g(\tau) \equiv \inf \{\theta g(y)+(1-\theta) g(z): \theta \in[0,1], y, z \in[a, b], \tau=\theta y+(1-\theta) z\} .
\end{gathered}
$$

If $s<0$, concave envelope of $\tilde{f}_{i}$ is considered.

For each $i$ and some small parameter $s$, the above system defines a continuous differentiable curve:

$$
\gamma: \tau \mapsto\left(u(\tau ; s, \bar{u}), v_{i}(\tau ; s, \bar{u}), \sigma_{i}(\tau ; s, \bar{u})\right), \quad \tau \in[0, s] \text { or }[s, 0],
$$


which is used to solve the Riemann problem. In the following, when there is no ambiguity, we may omit the dependency of $u, v_{i}, \sigma$ on $s, \bar{u}$. Also for later use, define $T_{s}^{i}[\bar{u}](\tau) \equiv u(\tau ; s, \bar{u}), \tau \in[0, s]$ or $[s, 0]$.

To solve the system 2.6), a general class of Lipschitz continuous curves is studied:

$$
\begin{gathered}
\Gamma_{i}(s, \bar{u})=\left\{\gamma: \gamma(\tau)=\left(u(\tau), v_{i}(\tau), \sigma_{i}(\tau)\right), \text { such that }|u(\tau)-\bar{u}|=|\tau|, v_{i}(0)=0,\right. \\
\left.\left|v_{i}(\tau)\right| \leq \delta_{1},\left|\sigma_{i}(\tau)-\lambda_{i}^{0}\right| \leq 2 C_{0} \delta_{1} \leq 1, \quad \tau \in[0, s] \text { or }[s, 0]\right\},
\end{gathered}
$$

for some small constant $0<\delta_{1} \ll 1$ and $C_{0}$ is defined in (2.5).

Definition $2.1([3, \sqrt{3}])$. Define the distance $D(\cdot, \cdot)$ in $\Gamma_{i}(s, \bar{u})$ by

$$
D\left(\gamma, \gamma^{\prime}\right)=\delta_{1}\left\|u-u^{\prime}\right\|_{L^{\infty}}+\left\|v_{i}-v_{i}^{\prime}\right\|_{L^{1}}+\left\|v_{i} \sigma_{i}-v_{i}^{\prime} \sigma_{i}^{\prime}\right\|_{L^{1}},
$$

where

$$
\gamma=\left(u, v_{i}, \sigma_{i}\right) \in \Gamma_{i}(s, \bar{u}), \gamma^{\prime}=\left(u^{\prime}, v_{i}^{\prime}, \sigma_{i}^{\prime}\right) \in \Gamma_{i}(s, \bar{u}) .
$$

And define the distance $P\left(\gamma ; \gamma^{\prime}\right)$ for $\Gamma_{i}(s, \bar{u})$ and $\Gamma_{i}^{\prime}\left(s^{\prime}, \bar{u}^{\prime}\right)\left(s s^{\prime} \geq 0\right)$ by

$$
P\left(\gamma ; \gamma^{\prime}\right)=D\left(\left.\gamma\right|_{I},\left.\gamma^{\prime}\right|_{I}\right)+\left|s-s^{\prime}\right|
$$

Here $\left.\gamma\right|_{I}$ is the restriction of $\gamma$ on the interval $I$ and $I$ is the common part of $[0, s]$ and $\left[0, s^{\prime}\right]$.

In 2, 3], it is proved that the map defined by the right hand side of 2.6] is a contraction map in $\Gamma_{i}(s, \bar{u})$. So 2.6 can be solved uniquely to define a curve $\gamma: \tau \mapsto\left(u(\tau), v_{i}(\tau), \sigma_{i}(\tau)\right)$. Based on this, the Riemann problem can be solved as follows.

Lemma 2.1. 3. 4] Let $A$ be a smooth, matrix valued map defined in a domain $\Omega \subset \mathbb{R}^{n} . \quad \gamma: \tau \mapsto\left(u(\tau), v_{i}(\tau), \sigma_{i}(\tau)\right)$ is the solution to 2.6 defined in a small neighborhood of zero. Define the right state $u^{+}=u(s)$. Then the unique vanishing viscosity solution of the Riemann problem (2.1) is the function

$$
u(x, t)=\left\{\begin{array}{lll}
u^{-}, & \text {if } & x / t<\sigma_{i}(0), \\
u(\tau), & \text { if } & x / t=\sigma_{i}(\tau), \\
u^{+}, & \text {if } & x / t>\sigma_{i}(s) .
\end{array}\right.
$$


On the other hand, any i-wave $\left(u_{l}, T_{s}^{i}\left[u_{l}\right](s)\right)$ can be associated with a curve $\gamma$ defined as the solution to 2.6 .

Remark 2.1. From the construction of the solution to the Riemann problem, it is easy to see that inside an i-wave, the speed $\sigma_{i}(\tau)$ is monotone increasing, since $\operatorname{conv}_{[0, s]} \tilde{f}_{i}$ is convex for $s>0$ while $\operatorname{conc}_{[s, 0]} \tilde{f}_{i}$ is concave for $s<0$. And when the assumption $(H)$ is imposed, the solution constructed can be reduced to the one given in [20, 22], which satisfies the Liu's entropy condition.

Definition 2.2. 2] Given two points $u_{0}$ and $u_{0}^{\prime}$, set $u_{1}=T_{s_{1}}^{i}\left[u_{0}\right]\left(s_{1}\right), u_{1}^{\prime}=$ $T_{s_{2}}^{i}\left[u_{0}^{\prime}\right]\left(s_{2}\right)$, for some $i \in\{1, \cdots, n\}$. Assume that $s_{1}$ is positive. Let $\tilde{f}_{1}$ be the scalar reduced flux function for (2.6) with initial data $u_{0}$ in $\left[0, s_{1}\right], \tilde{f}_{2}$ the reduced flux function in $\left[0, s_{2}\right]$ if $s_{2} \geq 0$ or in $\left[s_{2}, 0\right]$ if $s_{2}<0$ and with initial data $u_{0}^{\prime}$.

The amount of interaction $J_{i}$ for the $i$-waves $s_{1}, s_{2}$ is defined as follows:

1. if $s_{2} \geq 0$,

$$
\begin{aligned}
& J_{i}\left(s_{1}, s_{2}\right)=\int_{0}^{s_{1}}\left|\operatorname{conv}_{\left[0, s_{1}\right]} \tilde{f}_{1}(\xi)-\operatorname{conv}_{\left[0, s_{1}+s_{2}\right]}\left(\tilde{f}_{1} \cup \tilde{f}_{2}\right)(\xi)\right| \mathrm{d} \xi \\
& +\int_{s_{1}}^{s_{1}+s_{2}}\left|\operatorname{conv}_{\left[0, s_{2}\right]}\left(\tilde{f}_{1}\left(s_{1}\right)+\tilde{f}_{2}\left(\xi-s_{1}\right)\right)-\operatorname{conv}_{\left[0, s_{1}+s_{2}\right]}\left(\tilde{f}_{1} \cup \tilde{f}_{2}\right)(\xi)\right| \mathrm{d} \xi,
\end{aligned}
$$

where $\tilde{f}_{1} \cup \tilde{f}_{2}$ is the function defined in $\left[0, s_{1}+s_{2}\right]$ as

$$
\left(\tilde{f}_{1} \cup \tilde{f}_{2}\right)(s)= \begin{cases}\tilde{f}_{1}(s), & s \in\left[0, s_{1}\right], \\ \tilde{f}_{1}\left(s_{1}\right)+\tilde{f}_{2}\left(s-s_{1}\right), & s \in\left[s_{1}, s_{2}\right] ;\end{cases}
$$

2. if $-s_{1} \leq s_{2}<0$,

$$
\begin{aligned}
J_{i}\left(s_{1}, s_{2}\right)= & \int_{0}^{s_{1}+s_{2}}\left|\operatorname{conv}_{\left[0, s_{1}\right]} \tilde{f}_{1}(\xi)-\operatorname{conv}_{\left[0, s_{1}+s_{2}\right]} \tilde{f}_{1}(\xi)\right| \mathrm{d} \xi \\
& +\int_{s_{1}+s_{2}}^{s_{1}}\left|\operatorname{conv}_{\left[0, s_{1}\right]} \tilde{f}_{1}(\xi)-\operatorname{conc}_{\left[s_{1}+s_{2}, s_{1}\right]} \tilde{f}_{1}(\xi)\right| \mathrm{d} \xi
\end{aligned}
$$

3. if $s_{2}<-s_{1}$,

$$
\begin{aligned}
J_{i}\left(s_{1}, s_{2}\right)= & \int_{s_{1}+s_{2}}^{0}\left|\operatorname{conc}_{\left[s_{2}, 0\right]} \tilde{f}_{2}(\xi)-\operatorname{conc}_{\left[s_{2},-s_{1}\right]} \tilde{f}_{2}(\xi)\right| \mathrm{d} \xi \\
& +\int_{0}^{s_{1}}\left|\operatorname{conc}_{\left[s_{2}, 0\right]} \tilde{f}_{2}(\xi)-\operatorname{conv}_{\left[-s_{1}, 0\right]} \tilde{f}_{2}(\xi)\right| \mathrm{d} \xi .
\end{aligned}
$$




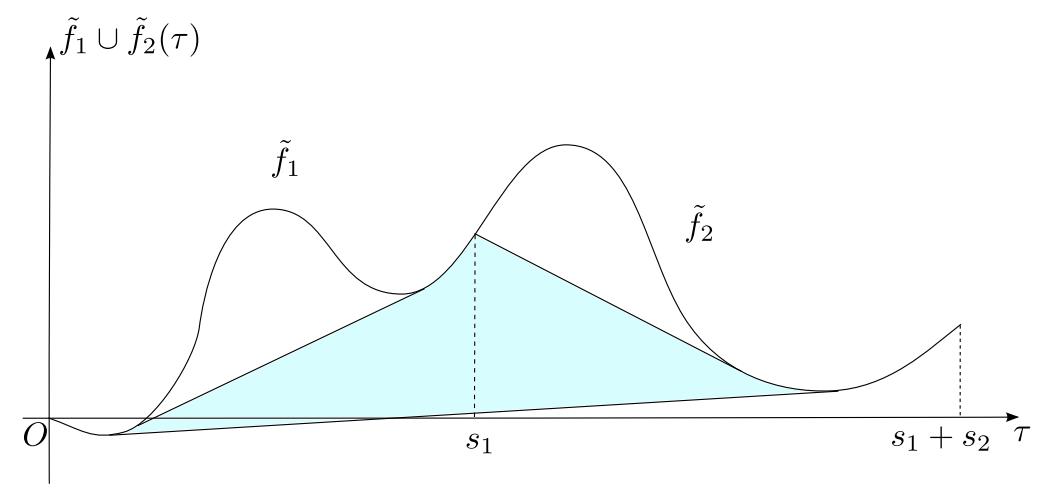

Figure 3: The amount of $J_{i}$ is represented in colored area, when $s_{1}, s_{2} \geq 0$

If $s_{1}<0$, then concave envelope is used instead of convex envelope in the above definition.

The amount of $J_{i}$ is in fact the area bounded by conv $\tilde{f}_{1}, \operatorname{conv} \tilde{f}_{2}$ and $\operatorname{conv}\left(\tilde{f}_{1} \cup\right.$ $\tilde{f}_{2}$ ). When $s_{1}, s_{2} \geq 0$, the area is illustrated in Figure 3 . For other cases, readers are referred to 2 .

In 2, the following Glimm type functional is defined.

$$
F_{o}(J) \equiv L(J)+M Q_{o}(J)
$$

where the subscript "o" is used in contrast to the new one which we will define later. In the above definition,

$$
\begin{aligned}
& Q_{o}(J)=Q_{d}(J)+\frac{1}{4} Q_{o s}(J), \\
& Q_{o s}(J)=\sum_{i=1}^{n} Q_{o s}^{i} \\
& Q_{o s}^{i}=\sum\left\{\int_{\substack{[0, \alpha] \\
\text { or }[\alpha, 0]}} \int_{\substack{[0, \beta] \\
\text { or }[\beta, 0]}}\left|\sigma_{\alpha}(\tau)-\sigma_{\beta}(\tau)\right| \mathrm{d} \tau \mathrm{d} \tau^{\prime}: \alpha \text { and } \beta \text { are i-waves crossing } \mathrm{J}\right\} .
\end{aligned}
$$

$L(J), Q_{d}$ are defined in (1.5). And $\sigma_{\alpha}(\tau) \equiv \sigma\left(\tau ; \alpha, u_{\alpha}\right)$ is the solution of 2.6 with initial state $u_{\alpha}$.

Remark 2.2. For two $i$-waves $\alpha, \beta$ with signed strengths also denoted by $\alpha$ and $\beta$ respectively, denote $\tilde{f}_{i}, \tilde{f}_{i}^{\prime}$ the corresponding reduced flux functions. Assume 
that $\alpha \beta>0$. For simplicity, suppose further that $\alpha>0$. Then we can define

$$
\begin{gathered}
s=\sup \left\{\tau \in[0, \alpha]: \operatorname{conv}_{[0, \alpha]} \tilde{f}_{i}(\xi)=\operatorname{conv}_{[0, \alpha+\beta]}\left(\tilde{f}_{i} \cup \tilde{f}_{i}^{\prime}\right)(\xi), \forall \xi \in[0, \tau]\right\}, \\
s^{\prime}=\inf \left\{\tau \in[0, \beta]: \operatorname{conv}_{[0, \beta]} \tilde{f}_{i}^{\prime}(\xi)=\operatorname{conv}_{[0, \alpha+\beta]}\left(\tilde{f}_{i} \cup \tilde{f}_{i}^{\prime}\right)(\alpha+\xi), \forall \xi \in[\tau, \beta]\right\} .
\end{gathered}
$$

We call $\tilde{Q}_{o s}^{i}(\alpha, \beta) \equiv Q_{o s}^{i}\left(\alpha-s, s^{\prime}\right)$ the quantity of effective interaction potential. By the definition of $J_{i}(\alpha, \beta)$, we can see that

$$
J_{i}(\alpha, \beta)=O(1) \tilde{Q}_{o s}^{i}(\alpha, \beta) .
$$

The functional $Q_{o}$ is non-increasing through the interaction. Indeed, the following lemma holds.

Lemma 2.2. Let $u_{l}, u_{m}$ and $u_{r}$ be three nearby states and let the Riemann problems $\left(u_{l}, u_{m}\right),\left(u_{m}, u_{r}\right)$ and $\left(u_{l}, u_{r}\right)$ be solved by waves $\alpha_{1}, \cdots, \alpha_{n}, \beta_{1}, \cdots, \beta_{n}$ and $\delta_{1}, \cdots, \delta_{n}$ respectively. The corresponding curves defined by 2.6 are $\gamma_{\alpha, i}, \gamma_{\beta, i}, \gamma_{\delta, i}$ $(i=1, \cdots, n)$. Denote the interaction potential before and after the interaction by $Q_{o}^{-}$and $Q_{o}^{+}$.

Then for some positive constant $c$,

$$
\begin{array}{r}
P\left(\gamma_{\alpha+\beta, i} ; \gamma_{\delta, i}\right)=O(1)\left\{\sum_{i>j}\left|\alpha_{i} \beta_{j}\right|+\sum_{i} J_{i}\right\}, \\
\sum_{i=1}^{n}\left|\delta_{i}-\left(\alpha_{i}+\beta_{i}\right)\right|=O(1)\left\{\sum_{i>j}\left|\alpha_{i} \beta_{j}\right|+\sum_{i} J_{i}\right\}, \\
\sum_{i=1}^{n}\left|\eta\left(\delta_{i}\right)-\eta\left(\alpha_{i}\right)-\eta\left(\beta_{i}\right)\right|=O(1)\left\{\sum_{i>j}\left|\alpha_{i} \beta_{j}\right|+\sum_{i} J_{i}\right\}, \\
\Delta Q_{o}=Q_{o}^{+}-Q_{o}^{-} \leq-c\left\{\sum_{i>j}\left|\alpha_{i} \beta_{j}\right|+\sum_{i} J_{i}\right\} .
\end{array}
$$

Here $\eta(\alpha)=\int_{0}^{\alpha} \sigma(\tau) \mathrm{d} \tau$ with $\sigma(\tau)=\sigma\left(\tau ; \alpha, u_{\alpha}\right)$ and $u_{\alpha}$ is the left state of the $i$-wave $\alpha$. And

$$
\begin{gathered}
\gamma_{\alpha+\beta, i}=\left\{\begin{array}{ll}
\gamma_{\alpha, i} \cup \gamma_{\beta, i}, & \alpha_{i}, \beta_{i} \geq 0, \\
\left.\gamma_{\alpha, i}\right|_{\left[0, \alpha_{i}+\beta_{i}\right]}, & -\alpha_{i} \leq \beta_{i}<0, \\
\left.\gamma_{\beta, i}\right|_{\left[\beta_{i}, \alpha_{i}+\beta_{i}\right]}, & \beta_{i} \leq-\alpha_{i}<0,
\end{array} \quad \text { for } \alpha_{i} \geq 0,\right. \\
\text { with } \gamma_{\alpha, i} \cup \gamma_{\beta, i}(\tau)= \begin{cases}\gamma_{\alpha, i}(\tau), & 0 \leq \tau \leq \alpha_{i}, \\
\gamma_{\alpha, i}\left(\alpha_{i}\right)+\gamma_{\beta, i}\left(\tau-\alpha_{i}\right), & \alpha_{i} \leq \tau \leq \alpha_{i}+\beta_{i} .\end{cases}
\end{gathered}
$$


A similar definition can be given for $\alpha_{i}<0$.

Proof. The estimates (2.13), 2.14 and 2.16) can be found in 2. We only prove 2.15 here.

Denote $\gamma(\tau)=\left(u(\tau), v_{i}(\tau), \sigma_{i}(\tau)\right), \gamma^{\prime}(\tau)=\left(u^{\prime}(\tau), v_{i}^{\prime}(\tau), \sigma_{i}^{\prime}(\tau)\right), \gamma^{\prime \prime}(\tau)=$ $\left(u^{\prime \prime}(\tau), v_{i}^{\prime \prime}(\tau), \sigma_{i}^{\prime \prime}(\tau)\right)$ the solutions to 2.6 corresponding to the i-waves $\delta_{i}, \alpha_{i}, \beta_{i}$ respectively. By the construction 2.6, we have

$$
\begin{gathered}
\eta\left(\delta_{i}\right)=\int_{0}^{\delta_{i}} \sigma_{i}(\tau) \mathrm{d} \tau=\tilde{f}_{i}\left(\delta_{i} ; \gamma\right)=\int_{0}^{\delta_{i}} \tilde{\lambda}_{i}\left(u(\tau), v_{i}(\tau), \sigma_{i}(\tau)\right) \mathrm{d} \tau, \\
\eta\left(\alpha_{i}\right)=\int_{0}^{\alpha_{i}} \sigma_{i}^{\prime}(\tau) \mathrm{d} \tau=\tilde{f}_{i}\left(\alpha_{i} ; \gamma^{\prime}\right)=\int_{0}^{\alpha_{i}} \tilde{\lambda}_{i}\left(u^{\prime}(\tau), v_{i}^{\prime}(\tau), \sigma_{i}^{\prime}(\tau)\right) \mathrm{d} \tau, \\
\eta\left(\beta_{i}\right)=\int_{0}^{\beta_{i}} \sigma_{i}^{\prime \prime}(\tau) \mathrm{d} \tau=\tilde{f}_{i}\left(\beta_{i} ; \gamma^{\prime \prime}\right)=\int_{0}^{\beta_{i}} \tilde{\lambda}_{i}\left(u^{\prime \prime}(\tau), v_{i}^{\prime \prime}(\tau), \sigma_{i}^{\prime \prime}(\tau)\right) \mathrm{d} \tau .
\end{gathered}
$$

It suffices to consider the case where $\alpha_{i}, \beta_{i}>0$. The other cases can be proved similarly. In this case, we have

$$
\begin{aligned}
& \left|\eta\left(\delta_{i}\right)-\eta\left(\alpha_{i}\right)-\eta\left(\beta_{i}\right)\right| \\
= & O(1)\left\{\sum_{i>j}\left|\alpha_{i} \beta_{j}\right|+\sum_{i} J_{i}\right\} \\
& +\int_{0}^{\alpha_{i}}\left[\tilde{\lambda}_{i}\left(u(\tau), v_{i}(\tau), \sigma_{i}(\tau)\right)-\tilde{\lambda}_{i}\left(u^{\prime}(\tau), v_{i}^{\prime}(\tau), \sigma_{i}^{\prime}(\tau)\right)\right] \mathrm{d} \tau \\
& +\int_{0}^{\beta_{i}}\left[\tilde{\lambda}_{i}\left(u\left(\alpha_{i}+\tau\right), v_{i}\left(\alpha_{i}+\tau\right), \sigma_{i}\left(\alpha_{i}+\tau\right)\right)-\tilde{\lambda}_{i}\left(u^{\prime \prime}(\tau), v_{i}^{\prime \prime}(\tau), \sigma_{i}^{\prime \prime}(\tau)\right)\right] \mathrm{d} \tau \\
= & O(1)\left\{\sum_{i>j}\left|\alpha_{i} \beta_{j}\right|+\sum_{i} J_{i}\right\}+I_{1}+I_{2} .
\end{aligned}
$$

By the property of $\tilde{\lambda}_{i}(2.5)$, the integral $I_{1}$ can be estimated as follows:

$$
\begin{aligned}
& I_{1} \leq O(1) \int_{0}^{\alpha_{i}}[\left\|D_{u} \tilde{\lambda}_{i}\left(u, v_{i}, \sigma_{i}\right)\right\|_{L_{\left[0, \alpha_{i}\right]}^{\infty}}\left|u(\tau)-u^{\prime}(\tau)\right| \\
&+\left\|D_{v_{i}} \tilde{\lambda}_{i}\left(u, v_{i}, \sigma_{i}\right)\right\|_{L_{\left[0, \alpha_{i}\right]}^{\infty}}\left|v_{i}(\tau)-v_{i}^{\prime}(\tau)\right| \\
&\left.+\left\|\frac{D_{\sigma_{i}} \tilde{\lambda}_{i}\left(u, v_{i}, \sigma_{i}\right)}{\left|v_{i}\right|}\right\|_{L_{\left[0, \alpha_{i}\right]}^{\infty}}\left|v_{i}(\tau) \| \sigma_{i}(\tau)-\sigma_{i}^{\prime}(\tau)\right|\right] \mathrm{d} \tau \\
& \leq O(1) \max \left\{\left|\alpha_{i}\right|, \bar{\delta}\right\}\left[\left\|u-u^{\prime}\right\|_{L_{\left[0, \alpha_{i}\right]}^{\infty}}+\left\|v_{i}-v_{i}^{\prime}\right\|_{\left.L_{\left[0, \alpha_{i}\right]}^{1}\right]}+\left\|v_{i} \sigma-v_{i}^{\prime} \sigma_{i}^{\prime}\right\|_{\left.L_{\left[0, \alpha_{i}\right]}^{1}\right]}\right],
\end{aligned}
$$


where $\bar{\delta}=\sup _{0 \leq \tau^{\prime} \leq \alpha_{i}, 0 \leq \tau^{\prime \prime} \leq \beta_{i}}\left\{\left|u^{\prime}\left(\tau^{\prime}\right)-u_{0}\right|,\left|u^{\prime \prime}\left(\tau^{\prime \prime}\right)-u_{0}\right|\right\}$. However, by 2.13 and the definition of the norm $P(\cdot ; \cdot)$, we have

$$
I_{1} \leq O(1)\left\{\sum_{i>j}\left|\alpha_{i} \beta_{j}\right|+\sum_{i} J_{i}\right\} .
$$

The bound of $I_{2}$ can be obtained similarly. So 2.15 holds.

Corollary 2.1. In the same setting as Lemma 2.2, for any constant $\bar{\sigma}$, the following holds when $\alpha_{i} \beta_{i}>0, \alpha_{k}=\beta_{k}=0(k \neq i)$ :

$$
\int_{0}^{\delta_{i}}\left|\sigma_{i}(\tau)-\bar{\sigma}\right| \mathrm{d} \tau-\int_{0}^{\alpha_{i}}\left|\sigma_{i}^{\prime}(\tau)-\bar{\sigma}\right| \mathrm{d} \tau-\int_{0}^{\beta_{i}}\left|\sigma_{i}^{\prime \prime}(\tau)-\bar{\sigma} \mathrm{d} \tau\right| \leq O(1) J_{i}
$$

Proof. It suffices to consider the case when $\delta_{i}$ is a single shock. If this is not the case, it is easy to see that either $\sigma_{i}(\tau)=\sigma_{i}^{\prime}(\tau)$ or $\sigma_{i}\left(\delta_{i}-\tau\right)=\sigma_{i}^{\prime}\left(\beta_{i}-\tau\right)$ outside the shock. Therefore, the contribution of these parts to both sides of 2.17) is zero.

From the monotonicity of $\sigma_{i}(\tau)$ and 2.15, we can compare $\sigma_{i}, \sigma_{i}^{\prime}$ and $\sigma_{i}^{\prime \prime}$ :

$$
\sigma_{i}^{\prime}(\tau) \geq \sigma_{i}(s)=\sigma_{i} \geq \sigma_{i}^{\prime \prime}\left(\tau^{\prime}\right), \text { for } \tau \in\left[0, \alpha_{i}\right], \quad \tau^{\prime} \in\left[0, \beta_{i}\right] .
$$

Now suppose that $\sigma_{i} \geq \bar{\sigma}$. The other cases can be treated similarly.

Since $\sigma_{i}^{\prime}(\tau) \geq \sigma_{i}(s) \geq \bar{\sigma}$, we have

$$
\begin{aligned}
& \int_{0}^{\delta_{i}}\left|\sigma_{i}(\tau)-\bar{\sigma}\right| \mathrm{d} \tau-\int_{0}^{\alpha_{i}}\left|\sigma_{i}^{\prime}(\tau)-\bar{\sigma}\right| \mathrm{d} \tau \\
= & \int_{0}^{\delta_{i}}\left(\sigma_{i}(\tau)-\bar{\sigma}\right) \mathrm{d} \tau-\int_{0}^{\alpha_{i}}\left(\sigma_{i}^{\prime}(\tau)-\bar{\sigma}\right) \mathrm{d} \tau \\
= & \eta\left(\delta_{i}\right)-\eta\left(\alpha_{i}\right)-\bar{\sigma}\left(\delta_{i}-\alpha_{i}\right) .
\end{aligned}
$$

By (2.14) and 2.15, the above equality can be estimated by

$$
\int_{0}^{\delta_{i}}\left|\sigma_{i}(\tau)-\bar{\sigma}\right| \mathrm{d} \tau-\int_{0}^{\alpha_{i}}\left|\sigma_{i}^{\prime}(\tau)-\bar{\sigma}\right| \mathrm{d} \tau=\eta\left(\beta_{i}\right)-\bar{\sigma} \beta_{i}+O(1) J_{i} .
$$

On the other hand,

$$
\eta\left(\beta_{i}\right)-\bar{\sigma} \beta_{i}=\int_{0}^{\beta_{i}}\left(\sigma_{i}^{\prime \prime}(\tau)-\bar{\sigma}\right) \mathrm{d} \tau \leq \int_{0}^{\beta_{i}}\left|\sigma_{i}^{\prime \prime}(\tau)-\bar{\sigma}\right| \mathrm{d} \tau .
$$

Hence, 2.17 follows. 
Another lemma is borrowed from [2, which is useful to estimate the difference of $\sigma$.

Lemma 2.3. Let $f, g$ be $C^{1}$ functions on the interval $[0, s]$. Then we have

$$
\left\|d\left(\operatorname{conv}_{[0, s]} f\right)-d\left(\operatorname{conv}_{[0, s]} g\right)\right\|_{L^{1}} \leq\|d f-d g\|_{L^{1}} .
$$

Inspired by the analysis in [14], we can improve the above interaction potential in a way similar to 1.6 . To do this, we define the following quantities for an i-wave $\left(\bar{u}, T_{s}^{i}[\bar{u}](s)\right)$. For any $\tau \in[0, s]$ or $[s, 0]$,

$$
\begin{aligned}
& {[\tau]_{s, \bar{u}, i}^{+}= \begin{cases}\max _{[a, b] \subset[0, s]}\left\{b: \tau \in[a, b], \text { such that } \frac{d}{d \xi} \sigma(\xi ; s, \bar{u})=0 \text { on }[a, b]\right\}, & s>0, \\
\min _{\substack{a, b] \subset[s, 0] \\
a<b}}\left\{a: \tau \in[a, b], \text { such that } \frac{d}{d \xi} \sigma(\xi ; s, \bar{u})=0 \text { on }[a, b]\right\}, & s<0,\end{cases} } \\
& {[\tau]_{s, \bar{u}, i}^{-}= \begin{cases}\min _{\substack{[a, b] \subset[0, s] \\
a<b}}\left\{a: \tau \in[a, b], \text { such that } \frac{d}{d \xi} \sigma(\xi ; s, \bar{u})=0 \text { on }[a, b]\right\}, & s>0, \\
\max _{\substack{[a, b] \subset[s, 0] \\
a<b}}\left\{b: \tau \in[a, b], \text { such that } \frac{d}{d \xi} \sigma(\xi ; s, \bar{u})=0 \text { on }[a, b]\right\}, & s<0 .\end{cases} }
\end{aligned}
$$

When there is no ambiguity, we may write $[\tau]^{ \pm}$to omit the dependency of $[\tau]^{ \pm}$ on $s, \bar{u}, i$. If $[\tau]^{-} \neq[\tau]^{+}$, then $\left(T_{s}^{i}[\bar{u}]\left([\tau]^{-}\right), T_{s}^{i}[\bar{u}]\left([\tau]^{+}\right)\right)$is a shock; while if $[\tau]^{-}=[\tau]^{+},\left(T_{s}^{i}[\bar{u}]\left([\tau]^{-}\right), T_{s}^{i}[\bar{u}]\left([\tau]^{+}\right)\right)$is just the state $T_{s}^{i}[\bar{u}]\left([\tau]^{-}\right)$.

Then we can define the quantity $t . v \cdot(\alpha, \beta)_{i}$ for general i-waves instead of only shocks under the same setting of Definition 2.2 .

$$
\text { t.v. }\left(T_{\alpha}^{i}\left[u_{\alpha}\right]\left(\tau_{1}\right), T_{\beta}^{i}\left[u_{\beta}\right]\left(\tau_{2}\right)\right)_{i} \equiv\left|\left[\tau_{1}\right]^{+}-\left[\tau_{1}\right]^{-}\right|+\left|\sum \gamma\right|+\left|\left[\tau_{2}\right]^{+}-\left[\tau_{2}\right]^{-}\right|,
$$

where the sum of the signed strength of $\gamma$ is over all the i-waves between $\left(T_{\alpha}^{i}\left[u_{\alpha}\right]\left(\left[\tau_{1}\right]^{-}\right), T_{\alpha}^{i}\left[u_{\alpha}\right]\left(\left[\tau_{1}\right]^{+}\right)\right)$and $\left(T_{\beta}^{i}\left[u_{\beta}\right]\left(\left[\tau_{2}\right]^{-}\right), T_{\beta}^{i}\left[u_{\beta}\right]\left(\left[\tau_{2}\right]^{+}\right)\right)$excluding the waves $\left(T_{\alpha}^{i}\left[u_{\alpha}\right]\left(\left[\tau_{1}\right]^{-}\right), T_{\alpha}^{i}\left[u_{\alpha}\right]\left(\left[\tau_{1}\right]^{+}\right)\right)$and $\left(T_{\beta}^{i}\left[u_{\beta}\right]\left(\left[\tau_{2}\right]^{-}\right), T_{\beta}^{i}\left[u_{\beta}\right]\left(\left[\tau_{2}\right]^{+}\right)\right)$.

With the above notations, we define $Q_{s}=\sum_{i} Q_{s}^{i}$ as follows:

$$
Q_{s}^{i}=\sum\left\{\int_{\substack{[0, \alpha] \\ \text { or }[\alpha, 0]}} \int_{\substack{[0, \beta] \\ \text { or }[\beta, 0]}} \frac{\left|\sigma_{\alpha}(\tau)-\sigma_{\beta}\left(\tau^{\prime}\right)\right| \mathrm{d} \tau \mathrm{d} \tau^{\prime}}{t . v \cdot\left(T_{\alpha}^{i}\left[u_{\alpha}\right](\tau), T_{\beta}^{i}\left[u_{\beta}\right]\left(\tau^{\prime}\right)\right)_{i}}: \alpha \text { and } \beta\right.
$$

are i-waves crossing $\mathrm{J}$ with left states $u_{\alpha}$ and $\left.u_{\beta}\right\}$.

$Q_{d}, L(J)$ and $M$ are defined as before.

Remark 2.3. From the Lipschitz continuity of the function $\sigma_{\alpha}(\tau)$, it is easy to see that

$$
Q_{s}^{i}(\alpha, \beta) \leq O(1)|\alpha \beta|
$$




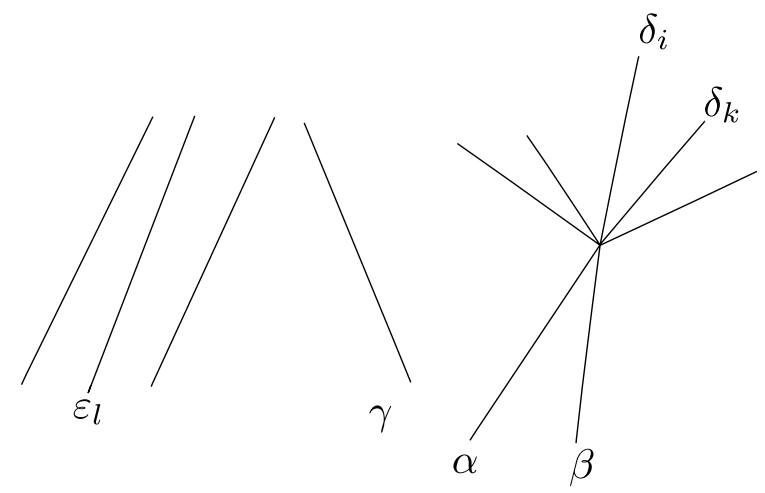

Figure 4: Case I

Now we are ready to prove Theorem 1.1 Theorem 1.1 is proved by considering some typical cases. In the first two cases, we assume that there is no shock splitting while the case with shock splitting will be discussed in the third case.

Proof of Theorem 1.1. Case (I)(cf. Fig 4$)$ : Suppose that $\alpha, \beta, \varepsilon_{l}(l=1,2,3, \cdots)$ are i-waves. $\gamma$ can be any other family wave. Note that there can be countably many waves in other families. Here we choose one $\gamma$ to represent them for simplicity. The following argument will be the same if there are more than one wave in other families. The total strength of all waves is bounded by Tot. Var. $u=O(1) L(t-)$. Assume $\alpha$ interacts with $\beta$ at time $t$ :

$$
\alpha+\beta \rightarrow \delta_{i}+\sum_{k \neq i} \delta_{k}
$$

Again, denote the left states of these i-waves $\alpha, \beta, \delta_{i}, \varepsilon_{l}$ by $u_{\alpha}, u_{\beta}, u_{\delta_{i}}, u_{\varepsilon_{l}}$ respectively. And for simplicity, we assume $\alpha \geq 0, \varepsilon_{l} \geq 0$.

Consider two subcases as follows.

Case (I.1) $\beta \geq 0$. Notice that in this case, there is no cancellation. Without loss of generality, we can assume that the $\mathrm{i}$-wave $\delta_{i}$ is a single shock. Then

$Q_{s}^{i}\left(\delta_{i}, \delta_{i}\right)=0, \frac{\partial}{\partial \tau}\left(t . v \cdot\left(T_{\varepsilon_{l}}^{i}\left[u_{\varepsilon_{l}}\right]\left(\tau^{\prime}\right), T_{\delta_{i}}^{i}\left[u_{\delta_{i}}\right](\tau)\right)_{i}\right)=0$, for $\tau \in\left[0, \delta_{i}\right], \tau^{\prime} \in\left[0, \varepsilon_{l}\right]$.

From the standard wave interaction estimate, we know that the change in the 
functionals $L$ and $Q_{d}$ at time $t$ are

$$
\begin{gathered}
\Delta L \equiv L(t+)-L(t-)=O(1) J_{i}(\alpha, \beta), \\
\Delta Q_{d} \equiv Q_{d}(t+)-Q_{d}(t-)=O(1)(L(t-)) J_{i}(\alpha, \beta) .
\end{gathered}
$$

And due to (2.23), we have

$$
\sum_{k \neq i} \Delta Q_{s}^{k} \equiv \sum_{k \neq i}\left(Q_{s}^{k}(t+)-Q_{s}^{k}(t-)\right)=O(1)(L(t-)) J_{i}(\alpha, \beta) .
$$

Before the wave interaction at time $t$, the wave interaction potential $Q_{s}$ is

$$
\begin{aligned}
& Q_{s}^{i}(t-)=2 \int_{[0, \alpha]} \int_{[0, \beta]} \frac{\left|\sigma_{\alpha}(\tau)-\sigma_{\beta}\left(\tau^{\prime}\right)\right| \mathrm{d} \tau \mathrm{d} \tau^{\prime}}{t \cdot v \cdot\left(T_{\alpha}^{i}\left[u_{\alpha}\right](\tau), T_{\beta}^{i}\left[u_{\beta}\right]\left(\tau^{\prime}\right)\right)_{i}} \\
& +2 \sum_{l}\left\{\int_{\left[0, \varepsilon_{l}\right]} \int_{[0, \alpha]} \frac{\left|\sigma_{\varepsilon_{l}}\left(\tau^{\prime}\right)-\sigma_{\alpha}(\tau)\right| \mathrm{d} \tau \mathrm{d} \tau^{\prime}}{t \cdot v \cdot\left(T_{\varepsilon_{l}}^{i}\left[u_{\varepsilon_{l}}\right]\left(\tau^{\prime}\right), T_{\alpha}^{i}\left[u_{\alpha}\right](\tau)\right)_{i}}+\int_{\left[0, \varepsilon_{l}\right]} \int_{[0, \beta]} \frac{\left|\sigma_{\varepsilon_{l}}\left(\tau^{\prime}\right)-\sigma_{\beta}(\tau)\right| \mathrm{d} \tau \mathrm{d} \tau^{\prime}}{t \cdot v \cdot\left(T_{\varepsilon_{l}}^{i}\left[u_{\varepsilon_{l}}\right]\left(\tau^{\prime}\right), T_{\beta}^{i}\left[u_{\beta}\right](\tau)\right)_{i}}\right\} \\
& +Q_{s}^{i}(\alpha, \alpha)+Q_{s}^{i}(\beta, \beta)+\sum_{l} Q_{s}^{i}\left(\varepsilon_{l}, \varepsilon_{l}\right) .
\end{aligned}
$$

After the wave interaction, it becomes

$$
Q_{s}^{i}(t+)=\sum_{l} 2 \int_{\left[0, \varepsilon_{l}\right]} \int_{\left[0, \delta_{i}\right]} \frac{\left|\sigma_{\varepsilon_{l}}\left(\tau^{\prime}\right)-\sigma_{\delta_{i}}(\tau)\right| \mathrm{d} \tau \mathrm{d} \tau^{\prime}}{t \cdot v \cdot\left(T_{\varepsilon_{l}}^{i}\left[u_{\varepsilon_{l}}\right]\left(\tau^{\prime}\right), T_{\delta_{i}}^{i}\left[u_{\delta_{i}}\right](\tau)\right)_{i}}+\sum_{l} Q_{s}^{i}\left(\varepsilon_{l}, \varepsilon_{l}\right) .
$$

Then

$$
\begin{aligned}
& \Delta Q_{s}^{i} \equiv Q_{s}^{i}(t+)-Q_{s}^{i}(t-) \\
= & -2 \int_{[0, \alpha]} \int_{[0, \beta]} \frac{\left|\sigma_{\alpha}(\tau)-\sigma_{\beta}\left(\tau^{\prime}\right)\right| \mathrm{d} \tau \mathrm{d} \tau^{\prime}}{t \cdot v \cdot\left(T_{\alpha}^{i}\left[u_{\alpha}\right](\tau), T_{\beta}^{i}\left[u_{\beta}\right]\left(\tau^{\prime}\right)\right)_{i}} \\
& +\sum_{l} 2\left\{\int_{\left[0, \varepsilon_{l}\right]} \int_{\left[0, \delta_{i}\right]} \frac{\left|\sigma_{\varepsilon_{l}}\left(\tau^{\prime}\right)-\sigma_{\delta_{i}}(\tau)\right| \mathrm{d} \tau \mathrm{d} \tau^{\prime}}{t \cdot v \cdot\left(T_{\varepsilon_{l}}^{i}\left[u_{\varepsilon_{l}}\right]\left(\tau^{\prime}\right), T_{\delta_{i}}^{i}\left[u_{\delta_{i}}\right](\tau)\right)_{i}}\right. \\
& \left.-\int_{\left[0, \varepsilon_{l}\right]} \int_{[0, \alpha]} \frac{\left|\sigma_{\varepsilon_{l}}\left(\tau^{\prime}\right)-\sigma_{\alpha}(\tau)\right| \mathrm{d} \tau \mathrm{d} \tau^{\prime}}{t \cdot v \cdot\left(T_{\varepsilon_{l}}^{i}\left[u_{\varepsilon_{l}}\right]\left(\tau^{\prime}\right), T_{\alpha}^{i}\left[u_{\alpha}\right](\tau)\right)_{i}}-\int_{\left[0, \varepsilon_{l}\right]} \int_{[0, \beta]} \frac{\left|\sigma_{\varepsilon_{l}}\left(\tau^{\prime}\right)-\sigma_{\beta}(\tau)\right| \mathrm{d} \tau \mathrm{d} \tau^{\prime}}{t \cdot v \cdot\left(T_{\varepsilon_{l}}^{i}\left[u_{\varepsilon_{l}}\right]\left(\tau^{\prime}\right), T_{\beta}^{i}\left[u_{\beta}\right](\tau)\right)_{i}}\right\} \\
& -\left\{Q_{s}^{i}(\alpha, \alpha)+Q_{s}^{i}(\beta, \beta)\right\} \\
\equiv & -2 Q_{s}^{i}(\alpha, \beta)+I+I I .
\end{aligned}
$$

By the definition of $t \cdot v \cdot(\cdot, \cdot)_{i}, 2.14$ and 2.24, we know that for any $l \in$ $\{1,2,3, \cdots\}$ and $\tau^{\prime} \in\left[0, \varepsilon_{l}\right]$,

$$
\begin{aligned}
& t . v \cdot\left(T_{\varepsilon_{l}}^{i}\left[u_{\varepsilon_{l}}\right]\left(\tau^{\prime}\right), T_{\delta_{i}}^{i}\left[u_{\delta_{i}}\right](\tau)\right)_{i}=t . v \cdot\left(T_{\varepsilon_{l}}^{i}\left[u_{\varepsilon_{l}}\right]\left(\tau^{\prime}\right), T_{\delta_{i}}^{i}\left[u_{\delta_{i}}\right]\left(\delta_{i}\right)\right)_{i} \\
= & t . v \cdot\left(T_{\varepsilon_{l}}^{i}\left[u_{\varepsilon_{l}}\right]\left(\tau^{\prime}\right), T_{\beta}^{i}\left[u_{\beta}\right](\beta)\right)_{i}+O(1) J_{i}(\alpha, \beta),
\end{aligned}
$$


t.v. $\left(T_{\varepsilon_{l}}^{i}\left[u_{\varepsilon_{l}}\right]\left(\tau^{\prime}\right), T_{\beta}^{i}\left[u_{\beta}\right](\beta)\right)_{i} \geq t . v .\left(T_{\varepsilon_{l}}^{i}\left[u_{\varepsilon_{l}}\right]\left(\tau^{\prime}\right), T_{\beta}^{i}\left[u_{\beta}\right](\tau)\right)_{i}$, for $\tau \in[0, \beta]$,

t.v. $\left(T_{\alpha}^{i}\left[u_{\alpha}\right](0), T_{\beta}^{i}\left[u_{\beta}\right](\beta)\right)_{i} \geq t . v \cdot\left(T_{\beta}^{i}\left[u_{\beta}\right]\left(\tau^{\prime \prime}\right), T_{\beta}^{i}\left[u_{\beta}\right](\tau)\right)_{i}$, for $\tau, \tau^{\prime \prime} \in[0, \beta]$,

t.v. $\left(T_{\varepsilon_{l}}^{i}\left[u_{\varepsilon_{l}}\right]\left(\tau^{\prime}\right), T_{\beta}^{i}\left[u_{\beta}\right](\beta)\right)_{i} \geq t . v .\left(T_{\varepsilon_{l}}^{i}\left[u_{\varepsilon_{l}}\right]\left(\tau^{\prime}\right), T_{\alpha}^{i}\left[u_{\alpha}\right](\tau)\right)_{i}$, for $\tau \in[0, \alpha]$,

t.v. $\left(T_{\alpha}^{i}\left[u_{\alpha}\right](0), T_{\beta}^{i}\left[u_{\beta}\right](\beta)\right)_{i} \geq t \cdot v \cdot\left(T_{\alpha}^{i}\left[u_{\alpha}\right]\left(\tau^{\prime \prime}\right), T_{\alpha}^{i}\left[u_{\alpha}\right](\tau)\right)_{i}$, for $\tau, \tau^{\prime \prime} \in[0, \alpha]$,

t.v. $\left(T_{\alpha}^{i}\left[u_{\alpha}\right](0), T_{\beta}^{i}\left[u_{\beta}\right](\beta)\right)_{i} \geq t . v .\left(T_{\alpha}^{i}\left[u_{\alpha}\right](\tau), T_{\beta}^{i}\left[u_{\beta}\right]\left(\tau^{\prime}\right)\right)_{i}$, for $\tau \in[0, \alpha], \tau^{\prime} \in[0, \beta]$,

and

$$
\text { t.v. }\left(T_{\varepsilon_{l}}^{i}\left[u_{\varepsilon_{l}}\right]\left(\tau^{\prime}\right), T_{\beta}^{i}\left[u_{\beta}\right](\beta)\right)_{i} \geq t . v \cdot\left(T_{\alpha}^{i}\left[u_{\alpha}\right](0), T_{\beta}^{i}\left[u_{\beta}\right](\beta)\right)_{i} .
$$

Notice that $(2.30)$ is true when there is no shock splitting.

Then by applying 2.30 and $2.31, I$ can be estimated as follows:

$$
\begin{aligned}
I \leq & \sum_{l} 2 \int_{\left[0, \varepsilon_{l}\right]} \mathrm{d} \tau^{\prime}\left\{\int_{\left[0, \delta_{i}\right]} \frac{\left|\sigma_{\varepsilon_{l}}\left(\tau^{\prime}\right)-\sigma_{\delta_{i}}(\tau)\right| \mathrm{d} \tau}{t \cdot v \cdot\left(T_{\varepsilon_{l}}^{i}\left[u_{\varepsilon_{l}}\right]\left(\tau^{\prime}\right), T_{\beta}^{i}\left[u_{\beta}\right](\beta)\right)_{i}+O(1) J_{i}(\alpha, \beta)}\right. \\
& \left.\quad-\int_{[0, \beta]} \frac{\left|\sigma_{\varepsilon_{l}}\left(\tau^{\prime}\right)-\sigma_{\beta}(\tau)\right| \mathrm{d} \tau}{t \cdot v \cdot\left(T_{\varepsilon_{l}}^{i}\left[u_{\varepsilon_{l}}\right]\left(\tau^{\prime}\right), T_{\beta}^{i}\left[u_{\beta}\right](\beta)\right)_{i}}-\int_{[0, \alpha]} \frac{\left|\sigma_{\varepsilon_{l}}\left(\tau^{\prime}\right)-\sigma_{\alpha}(\tau)\right| \mathrm{d} \tau}{t \cdot v \cdot\left(T_{\varepsilon_{l}}^{i}\left[u_{\varepsilon_{l}}\right]\left(\tau^{\prime}\right), T_{\beta}^{i}\left[u_{\beta}\right](\beta)\right)_{i}}\right\} \\
& +\sum_{l} 2\left\{\int_{\left[0, \varepsilon_{l}\right]} \int_{[0, \alpha]} \frac{\left|\sigma_{\varepsilon_{l}}\left(\tau^{\prime}\right)-\sigma_{\alpha}(\tau)\right| \mathrm{d} \tau \mathrm{d} \tau^{\prime}}{t \cdot v \cdot\left(T_{\varepsilon_{l}}^{i}\left[u_{\varepsilon_{l}}\right]\left(\tau^{\prime}\right), T_{\beta}^{i}\left[u_{\beta}\right](\beta)\right)_{i}}-\int_{\left[0, \varepsilon_{l}\right]} \int_{[0, \alpha]} \frac{\left|\sigma_{\varepsilon_{l}}\left(\tau^{\prime}\right)-\sigma_{\alpha}(\tau)\right| \mathrm{d} \tau \mathrm{d} \tau^{\prime}}{t \cdot v \cdot\left(T_{\varepsilon_{l}}^{i}\left[u_{\varepsilon_{l}}\right]\left(\tau^{\prime}\right), T_{\alpha}^{i}\left[u_{\alpha}\right](\tau)\right)_{i}}\right\} \\
= & \sum_{l} 2 \int_{0}^{\varepsilon_{l}} \mathrm{~d} \tau^{\prime} \frac{\int_{0}^{\delta_{i}}\left|\sigma_{\varepsilon_{l}}\left(\tau^{\prime}\right)-\sigma_{\delta_{i}}(\tau)\right| \mathrm{d} \tau-\int_{0}^{\beta}\left|\sigma_{\varepsilon_{l}}\left(\tau^{\prime}\right)-\sigma_{\beta}(\tau)\right| \mathrm{d} \tau-\int_{0}^{\alpha}\left|\sigma_{\varepsilon_{l}}\left(\tau^{\prime}\right)-\sigma_{\alpha}(\tau)\right| \mathrm{d} \tau}{t \cdot v \cdot\left(T_{\varepsilon_{l}}^{i}\left[u_{\varepsilon_{l}}\right]\left(\tau^{\prime}\right), T_{\beta}^{i}\left[u_{\beta}\right](\beta)\right)_{i}} \\
& +\sum_{l} 2\left\{\int_{\left[0, \varepsilon_{l}\right]} \int_{[0, \alpha]} \frac{\left|\sigma_{\varepsilon_{l}}\left(\tau^{\prime}\right)-\sigma_{\alpha}(\tau)\right| \mathrm{d} \tau \mathrm{d} \tau^{\prime}}{t \cdot v \cdot\left(T_{\varepsilon_{l}}^{i}\left[u_{\left.\varepsilon_{l}\right]}\right]\left(\tau^{\prime}\right), T_{\beta}^{i}\left[u_{\beta}\right](\beta)\right)_{i}}-\int_{\left[0, \varepsilon_{l}\right]} \int_{[0, \alpha]} \frac{\left|\sigma_{\varepsilon_{l}}\left(\tau^{\prime}\right)-\sigma_{\alpha}(\tau)\right| \mathrm{d} \tau \mathrm{d} \tau^{\prime}}{t \cdot v \cdot\left(T_{\varepsilon_{l}}^{i}\left[u_{\varepsilon_{l}}\right]\left(\tau^{\prime}\right), T_{\alpha}^{i}\left[u_{\alpha}\right](\tau)\right)_{i}}\right\} \\
& +O(1)(L(t-)) J_{i}(\alpha, \beta) \\
\equiv & I_{1}+I_{2}+O(1)(L(t-)) J_{i}(\alpha, \beta) .
\end{aligned}
$$

The estimate of $I_{2}$ is simple because it is always negative due to 2.33). And 
from Corollary 2.1.

$$
I_{1} \leq O(1) \sum_{l} \frac{\left|\varepsilon_{l}\right| J_{i}(\alpha, \beta)}{t . v \cdot\left(T_{\alpha}^{i}\left[u_{\alpha}\right](0), T_{\beta}^{i}\left[u_{\beta}\right](\beta)\right)_{i}} \leq O(1)(L(t-)) Q_{s}^{i}(\alpha, \beta) .
$$

Here we have used 2.36 and 2.12).

On the other hand, $I I \leq 0$.

Therefore,

$$
\begin{aligned}
\Delta Q_{s}^{i} & =-2 Q_{s}^{i}(\alpha, \beta)+I+I I \\
& \leq-2 Q_{s}^{i}(\alpha, \beta)+O(1)(L(t-)) Q_{s}^{i}(\alpha, \beta) .
\end{aligned}
$$

By combining the estimates 2.25, 2.26), 2.27) and 2.38), we can get (1.7) for some suitably chosen constant $M$ when the total variation of waves is sufficiently small.

Case (I.2) $\beta<0$. In addition, we assume that $\delta_{i}>0$ and $|\alpha|>|\beta|$. The case when $\delta_{i} \leq 0$ can be treated similarly.

Then in this case, the amount of cancellation is $C(\alpha, \beta)=|\beta|$. By the Lipschitz continuity of the wave curve, it is straightforward to get

$$
\begin{gathered}
\Delta L \equiv L(t+)-L(t-)=-C(\alpha, \beta), \\
\Delta Q_{d} \equiv Q_{d}(t+)-Q_{d}(t-)=O(1)(L(t-)) C(\alpha, \beta), \\
\sum_{k \neq i} \Delta Q_{s}^{k} \equiv \sum_{k \neq i}\left(Q_{s}^{k}(t+)-Q_{s}^{k}(t-)\right)=O(1)(L(t-)) C(\alpha, \beta) .
\end{gathered}
$$

Similar to the previous case, we have

$$
\begin{aligned}
\Delta & Q_{s}^{i} \equiv Q_{s}^{i}(t+)-Q_{s}^{i}(t-) \\
= & -2 \int_{[0, \alpha]} \int_{[\beta, 0]} \frac{\left|\sigma_{\alpha}(\tau)-\sigma_{\beta}\left(\tau^{\prime}\right)\right| \mathrm{d} \tau \mathrm{d} \tau^{\prime}}{t \cdot v \cdot\left(T_{\alpha}^{i}\left[u_{\alpha}\right](\tau), T_{\beta}^{i}\left[u_{\beta}\right]\left(\tau^{\prime}\right)\right)_{i}} \\
& +\sum_{l} 2\left\{\int_{\left[0, \varepsilon_{l}\right]} \int_{\left[0, \delta_{i}\right]} \frac{\left|\sigma_{\varepsilon_{l}}\left(\tau^{\prime}\right)-\sigma_{\delta_{i}}(\tau)\right| \mathrm{d} \tau \mathrm{d} \tau^{\prime}}{t \cdot v \cdot\left(T_{\varepsilon_{l}}^{i}\left[u_{\varepsilon_{l}}\right]\left(\tau^{\prime}\right), T_{\delta_{i}}^{i}\left[u_{\delta_{i}}\right](\tau)\right)_{i}}\right. \\
& \left.-\int_{\left[0, \varepsilon_{l}\right]} \int_{[0, \alpha]} \frac{\left|\sigma_{\varepsilon_{l}}\left(\tau^{\prime}\right)-\sigma_{\alpha}(\tau)\right| \mathrm{d} \tau \mathrm{d} \tau^{\prime}}{t \cdot v \cdot\left(T_{\varepsilon_{l}}^{i}\left[u_{\varepsilon_{l}}\right]\left(\tau^{\prime}\right), T_{\alpha}^{i}\left[u_{\alpha}\right](\tau)\right)_{i}}-\int_{\left[0, \varepsilon_{l}\right]} \int_{[\beta, 0]} \frac{\left|\sigma_{\varepsilon_{l}}\left(\tau^{\prime}\right)-\sigma_{\beta}(\tau)\right| \mathrm{d} \tau \mathrm{d} \tau^{\prime}}{t . v \cdot\left(T_{\varepsilon_{l}}^{i}\left[u_{\varepsilon_{l}}\right]\left(\tau^{\prime}\right), T_{\beta}^{i}\left[u_{\beta}\right](\tau)\right)_{i}}\right\} \\
& +\left\{Q_{s}^{i}\left(\delta_{i}, \delta_{i}\right)-Q_{s}^{i}(\alpha, \alpha)-Q_{s}^{i}(\beta, \beta)\right\} \\
\equiv & -2 Q_{s}^{i}(\alpha, \beta)+I+I I .
\end{aligned}
$$

By the Lipschitz continuity of $\sigma(\tau ; s, u)$ on $s$, we have

$$
\left|\sigma_{\delta_{i}}(\tau)-\sigma_{\alpha}(\tau)\right| \leq O(1) C(\alpha, \beta) .
$$



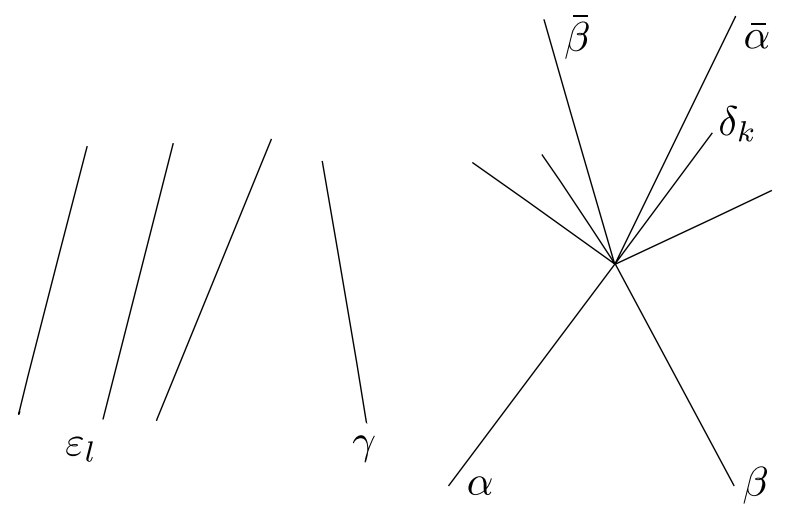

Figure 5: Case II

Since there is no shock splitting, then for $\tau \in\left[0, \delta_{i}\right]$,

$$
\left|t . v .\left(T_{\varepsilon_{l}}^{i}\left[u_{\varepsilon_{l}}\right]\left(\tau^{\prime}\right), T_{\delta_{i}}^{i}\left[u_{\delta_{i}}\right](\tau)\right)_{i}-t . v \cdot\left(T_{\varepsilon_{l}}^{i}\left[u_{\varepsilon_{l}}\right]\left(\tau^{\prime}\right), T_{\alpha}^{i}\left[u_{\alpha}\right](\tau)\right)_{i}\right| \leq O(1) C(\alpha, \beta) .
$$

Thus

$$
\begin{aligned}
I= & \sum_{l} 2\left\{\int_{\left[0, \varepsilon_{l}\right]} \int_{\left[0, \delta_{i}\right]}\left[\frac{\left|\sigma_{\varepsilon_{l}}\left(\tau^{\prime}\right)-\sigma_{\delta_{i}}(\tau)\right| \mathrm{d} \tau \mathrm{d} \tau^{\prime}}{t \cdot v \cdot\left(T_{\varepsilon_{l}}^{i}\left[u_{\varepsilon_{l}}\right]\left(\tau^{\prime}\right), T_{\delta_{i}}^{i}\left[u_{\delta_{i}}\right](\tau)\right)_{i}}-\frac{\left|\sigma_{\varepsilon_{l}}\left(\tau^{\prime}\right)-\sigma_{\alpha}(\tau)\right| \mathrm{d} \tau \mathrm{d} \tau^{\prime}}{t \cdot v \cdot\left(T_{\varepsilon_{l}}^{i}\left[u_{\varepsilon_{l}}\right]\left(\tau^{\prime}\right), T_{\alpha}^{i}\left[u_{\alpha}\right](\tau)\right)_{i}}\right]\right. \\
& \left.-\int_{\left[0, \varepsilon_{l}\right]}\left[\int_{\left[\delta_{i}, \alpha\right]} \frac{\left|\sigma_{\varepsilon_{l}}\left(\tau^{\prime}\right)-\sigma_{\alpha}(\tau)\right| \mathrm{d} \tau \mathrm{d} \tau^{\prime}}{t \cdot v \cdot\left(T_{\varepsilon_{l}}^{i}\left[u_{\varepsilon_{l}}\right]\left(\tau^{\prime}\right), T_{\alpha}^{i}\left[u_{\alpha}\right](\tau)\right)_{i}}+\int_{[\beta, 0]} \frac{\left|\sigma_{\varepsilon_{l}}\left(\tau^{\prime}\right)-\sigma_{\beta}(\tau)\right| \mathrm{d} \tau \mathrm{d} \tau^{\prime}}{t \cdot v \cdot\left(T_{\varepsilon_{l}}^{i}\left[u_{\varepsilon_{l}}\right]\left(\tau^{\prime}\right), T_{\beta}^{i}\left[u_{\beta}\right](\tau)\right)_{i}}\right]\right\} \\
& \leq O(1)(L(t-)) C(\alpha, \beta),
\end{aligned}
$$

where we have used (2.23) and (2.42). Similar argument can be applied to $I I$. Thus,

$$
\Delta Q_{s}^{i} \leq O(1)(L(t-)) C(\alpha, \beta) .
$$

1.7 holds by choosing a suitably large constant $M$ when the total variation of the solution is small.

Case (II)(cf. Fig.5): Assume that $\varepsilon_{l}(l=1,2,3, \cdots), \alpha$ are i-waves and $\beta$ is a $\mathrm{j}$-wave $(i>j)$. $\gamma$ can be any other k-family wave $(k \neq i)$. The interaction is at time $t$

$$
\alpha+\beta \longrightarrow \bar{\alpha}+\bar{\beta}+\sum_{k \neq i, j} \delta_{k}
$$

For simplicity, we may also assume $\alpha, \varepsilon_{l} \geq 0$. 
From the standard wave interaction estimate 2.14) and (2.23), we know the changes in the functionals $L$ and $Q_{d}$ at time $t$ are

$$
\begin{gathered}
\Delta L \equiv L(t+)-L(t-)=O(1)|\alpha \beta|, \\
\Delta Q_{d} \equiv Q_{d}(t+)-Q_{d}(t-)=-|\alpha \beta|+O(1)(L(t-))|\alpha \beta|, \\
\sum_{k \neq i} \Delta Q_{s}^{k} \equiv \sum_{k \neq i}\left(Q_{s}^{k}(t+)-Q_{s}^{k}(t-)\right)=O(1)(L(t-))|\alpha \beta| .
\end{gathered}
$$

And

$$
\begin{aligned}
& \Delta Q_{s}^{i} \equiv Q_{s}^{i}(t+)-Q_{s}^{i}(t-) \\
= & \sum_{l} 2\left\{Q_{s}^{i}\left(\varepsilon_{l}, \bar{\alpha}\right)-Q_{s}^{i}\left(\varepsilon_{l}, \alpha\right)\right\}+Q_{s}^{i}(\bar{\alpha}, \bar{\alpha})-Q_{s}^{i}(\alpha, \alpha) \\
= & \sum_{l} 2\left\{\int_{\left[0, \varepsilon_{l}\right]} \int_{[0, \bar{\alpha}]} \frac{\left|\sigma_{\varepsilon_{l}}\left(\tau^{\prime}\right)-\sigma_{\bar{\alpha}}(\tau)\right| \mathrm{d} \tau \mathrm{d} \tau^{\prime}}{t \cdot v \cdot\left(T_{\varepsilon_{l}}^{i}\left[u_{\varepsilon_{l}}\right]\left(\tau^{\prime}\right), T_{\bar{\alpha}}^{i}\left[u_{\bar{\alpha}}\right](\tau)\right)_{i}}-\int_{\left[0, \varepsilon_{l}\right]} \int_{[0, \alpha]} \frac{\left|\sigma_{\varepsilon_{l}}\left(\tau^{\prime}\right)-\sigma_{\alpha}(\tau)\right| \mathrm{d} \tau \mathrm{d} \tau^{\prime}}{t \cdot v \cdot\left(T_{\varepsilon_{l}}^{i}\left[u_{\varepsilon_{l}}\right]\left(\tau^{\prime}\right), T_{\alpha}^{i}\left[u_{\alpha}\right](\tau)\right)_{i}}\right\} \\
& +\left\{Q_{s}^{i}(\bar{\alpha}, \bar{\alpha})-Q_{s}^{i}(\alpha, \alpha)\right\} .
\end{aligned}
$$

It is easy to verify the following inequality for any constant $\bar{\sigma}$ :

$$
\begin{aligned}
& \left|\int_{[0, \bar{\alpha}]}\right| \bar{\sigma}-\sigma_{\bar{\alpha}}(\tau)\left|\mathrm{d} \tau-\int_{[0, \alpha]}\right| \bar{\sigma}-\sigma_{\alpha}(\tau)|\mathrm{d} \tau| \\
\leq & \int_{0}^{\min \{\bar{\alpha}, \alpha\}}\left|\sigma_{\alpha}(\tau)-\sigma_{\bar{\alpha}}(\tau)\right| \mathrm{d} \tau+O(1)|\bar{\alpha}-\alpha| .
\end{aligned}
$$

In addition, by applying Lemma 2.3 and the similar argument in proving (2.15, we have

$$
\begin{aligned}
& \int_{0}^{\min \{\bar{\alpha}, \alpha\}}\left|\sigma_{\alpha}(\tau)-\sigma_{\bar{\alpha}}(\tau)\right| \mathrm{d} \tau \\
& \leq\left\|d \tilde{f}_{i}\left(\tau ; \gamma_{\bar{\alpha}}\right)-d \tilde{f}_{i}\left(\tau ; \gamma_{\alpha}\right)\right\|_{L_{\min \{\bar{\alpha}, \alpha\}}^{1}} \leq O(1) D\left(\gamma_{\bar{\alpha}}, \gamma_{\alpha}\right) .
\end{aligned}
$$

Here $\gamma_{\alpha}, \gamma_{\bar{\alpha}}$ are the solutions to 2.6 corresponding to $\alpha, \bar{\alpha}$. Then by 2.14, we can conclude that for any $l \in 1,2,3, \cdots$,

$$
\left|\int_{[0, \bar{\alpha}]}\right| \sigma_{\varepsilon_{l}}\left(\tau^{\prime}\right)-\sigma_{\bar{\alpha}}(\tau)\left|\mathrm{d} \tau-\int_{[0, \alpha]}\right| \sigma_{\varepsilon_{l}}\left(\tau^{\prime}\right)-\sigma_{\alpha}(\tau)|\mathrm{d} \tau| \leq O(1)|\alpha \beta| .
$$

On the other hand, when there is no shock splitting, we have

$$
\left|t . v .\left(T_{\varepsilon_{l}}^{i}\left[u_{\varepsilon_{l}}\right]\left(\tau^{\prime}\right), T_{\alpha}^{i}\left[u_{\alpha}\right](\tau)\right)_{i}-t . v \cdot\left(T_{\varepsilon_{l}}^{i}\left[u_{\varepsilon_{l}}\right]\left(\tau^{\prime}\right), T_{\bar{\alpha}}^{i}\left[u_{\bar{\alpha}}\right](\tau)\right)_{i}\right|=O(1)|\alpha \beta| .
$$




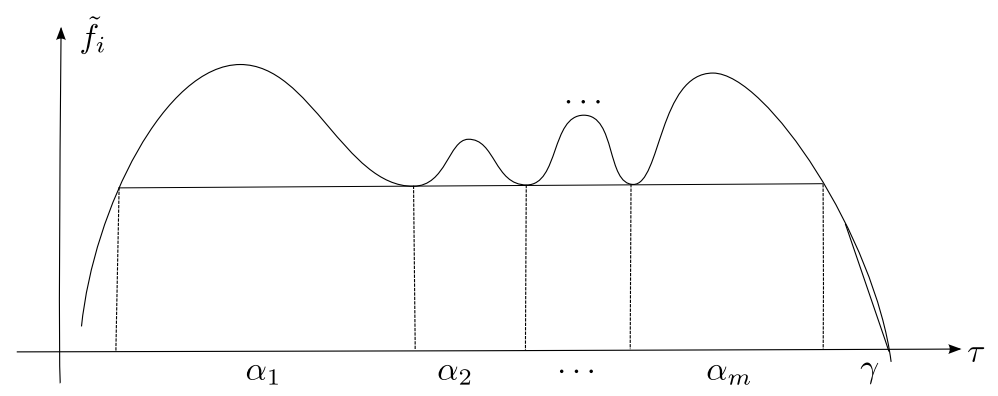

Figure 6: A shock splits into several shocks

Thus,

$$
\Delta Q_{s}^{i} \leq O(1)(L(t-))|\alpha \beta|
$$

By combining 2.45, 2.46, 2.47) and 2.52, we can get (1.7).

Case (III) In this case, we consider the case when shock splitting happens. In fact, when the characteristic field is not genuinely linear, a shock may split into several small shocks, cf. Figure 6 through the wave interaction, in particular, through wave cancellation.

Since $\operatorname{conv}_{\left[0, \alpha_{1}+\cdots+\alpha_{m}\right]} \tilde{f}_{i}(\tau)=\operatorname{conv}_{\left[0, \alpha_{1}+\cdots+\alpha_{m-1}\right]} \tilde{f}_{i}\left(\tau^{\prime}\right)=\operatorname{conv}_{\left[0, \alpha_{m}\right]} \tilde{f}_{i}\left(\tau^{\prime \prime}\right)$, the large shock with strength $\alpha_{1}+\cdots+\alpha_{m}$ may split into two shocks with strength $\alpha_{1}+\cdots+\alpha_{m-1}$ and $\alpha_{m}$ :

$$
\left\lfloor\alpha_{1}+\cdots+\alpha_{m}\right\rfloor \rightarrow\left\lfloor\alpha_{1}+\cdots+\alpha_{m-1}\right\rfloor+\alpha_{m}
$$

where $\left\lfloor\alpha_{1}+\cdots+\alpha_{m}\right\rfloor$ is a shock consisting of $m$ parts from left to right denoted by $\alpha_{i}, i=1, \cdots, m$ as shown in Figure 6 . This could happen for example when there is an i-wave $\beta$ on the right of $\left\lfloor\alpha_{1}+\cdots+\alpha_{m}\right\rfloor$ with $-\alpha_{m} \leq \beta \leq 0$ that cancels part of $\alpha_{m}$. Then the interaction:

$$
\left\lfloor\alpha_{1}+\cdots+\alpha_{m}\right\rfloor+\beta \rightarrow\left\lfloor\alpha_{1}+\cdots+\alpha_{m-1}\right\rfloor+\left\lfloor\alpha_{m}+\beta\right\rfloor
$$

is equivalent to a shock splitting and an interaction

$$
\begin{gathered}
\left\lfloor\alpha_{1}+\cdots+\alpha_{m}\right\rfloor \rightarrow\left\lfloor\alpha_{1}+\cdots+\alpha_{m-1}\right\rfloor+\alpha_{m}, \\
\alpha_{m}+\beta \rightarrow\left\lfloor\alpha_{m}+\beta\right\rfloor .
\end{gathered}
$$

The splitting of a shock is the consequence of cancellation, however, the amount of cancellation can be arbitrarily small. 
Observe that the change of the speed of the shock through the splitting is zero: $\sigma_{\left\lfloor\alpha_{1}+\cdots+\alpha_{m}\right\rfloor}=\sigma_{\left\lfloor\alpha_{1}+\cdots+\alpha_{m-1}\right\rfloor}=\cdots=\sigma_{\alpha_{1}}=\cdots=\sigma_{\alpha_{m}}=\bar{\sigma}$. Then the splitting of the i-th shock $\left\lfloor\alpha_{1}+\cdots+\alpha_{m}\right\rfloor$ into $\left\lfloor\alpha_{1}+\cdots+\alpha_{m-1}\right\rfloor$ and $\alpha_{m}$ makes the functional $Q_{s}^{i}$ increase by amount

$$
\begin{aligned}
& Q_{s}^{i}\left(\left\lfloor\alpha_{1}+\cdots+\alpha_{m-1}\right\rfloor, \gamma\right)+Q_{s}^{i}\left(\alpha_{1}, \gamma\right)-Q_{s}^{i}\left(\left\lfloor\alpha_{1}+\cdots+\alpha_{m}\right\rfloor, \gamma\right) \\
= & \int_{\substack{[0, \gamma] \\
\text { or }[\gamma, 0]}}\left(\frac{1}{t . v \cdot\left(\alpha_{m}, T_{\gamma}^{i}\left[u_{\gamma}\right](s)\right)_{i}}-\frac{1}{t . v \cdot\left(\left\lfloor\alpha_{1}+\cdots+\alpha_{m}\right\rfloor, T_{\gamma}^{i}\left[u_{\gamma}\right](s)\right)_{i}}\right)\left|\alpha_{m}\right|\left|\bar{\sigma}-\sigma_{\gamma}(s)\right| \mathrm{d} s,
\end{aligned}
$$

when there is an i-th shock $\gamma$ on the right of $\left\lfloor\alpha_{1}+\cdots+\alpha_{m}\right\rfloor$.

To understand the effect of the shock splitting on the functional $Q_{s}$, we consider the following typical subcases.

Subcase (III.1): We first consider a series of splittings in the time interval $[0, T]:$

$$
\begin{gathered}
\left\lfloor\alpha_{1}+\cdots+\alpha_{m}\right\rfloor \rightarrow\left\lfloor\alpha_{1}+\cdots+\alpha_{m-2}\right\rfloor+\left\lfloor\alpha_{m-1}+\alpha_{m}\right\rfloor \\
\rightarrow\left\lfloor\alpha_{1}+\cdots+\alpha_{m-3}\right\rfloor+\alpha_{m-2}+\alpha_{m-1}+\alpha_{m} \\
\cdots \\
\cdots \\
\rightarrow \alpha_{1}+\alpha_{2}+\cdots+\alpha_{m} .
\end{gathered}
$$

And assume for the moment that there are no other wave interactions in the meanwhile. In this case, the change of the functional $Q_{s}^{i}$ is

$$
\begin{aligned}
& \frac{1}{2} \Delta Q_{s}^{i}=\sum_{k=1}^{m} \sum_{\gamma} Q_{s}^{i}\left(\alpha_{k}, \gamma\right)-\sum_{\gamma} Q_{s}^{i}\left(\left\lfloor\alpha_{1}+\alpha_{2}+\cdots+\alpha_{m}\right\rfloor, \gamma\right) \\
= & \sum_{k=1}^{m} \sum_{\gamma}\left\{\int_{\substack{[0, \gamma] \\
\text { or }[\gamma, 0]}} \mathrm{d} s\left(\frac{\left|\alpha_{k}\right|\left|\bar{\sigma}-\sigma_{\gamma}(s)\right|}{t \cdot v \cdot\left(\alpha_{k}, T_{\gamma}^{i}\left[u_{\gamma}\right](s)\right)_{i}}-\frac{\left|\alpha_{k}\right|\left|\bar{\sigma}-\sigma_{\gamma}(s)\right|}{t \cdot v \cdot\left(\left\lfloor\sum_{l=1}^{m} \alpha_{l}\right\rfloor, T_{\gamma}^{i}\left[u_{\gamma}\right](s)\right)_{i}}\right)\right\} \\
= & \sum_{k=1}^{m} \sum_{\gamma}\left\{\int_{\substack{[0, \gamma] \\
\text { or }[\gamma, 0]}}\left(\frac{\left(\sum_{l=1}^{m} \alpha_{l}-\alpha_{k}\right)\left|\alpha_{k}\right|\left|\bar{\sigma}-\sigma_{\gamma}(s)\right|}{\left[t \cdot v \cdot\left(\alpha_{k}, T_{\gamma}^{i}\left[u_{\gamma}\right](s)\right)_{i}\right]\left[t \cdot v \cdot\left(\left\lfloor\sum_{l=1}^{m} \alpha_{l}\right\rfloor, T_{\gamma}^{i}\left[u_{\gamma}\right](s)\right)_{i}\right]}\right) \mathrm{d} s\right\} \\
\leq & \sum_{k=1}^{m} \sum_{\gamma}\left|\alpha_{k}\right||\gamma| .
\end{aligned}
$$


Here, we have used

$$
\begin{gathered}
\left|\bar{\sigma}-\sigma_{\gamma}(s)\right| \leq t \cdot v \cdot\left(\alpha_{k}, T_{\gamma}^{i}\left[u_{\gamma}\right](s)\right)_{i}, \\
0 \leq\left(\sum_{l=1}^{m} \alpha_{l}-\alpha_{k}\right) \leq t . v \cdot\left(\left\lfloor\sum_{l=1}^{m} \alpha_{l}\right\rfloor, T_{\gamma}^{i}\left[u_{\gamma}\right](s)\right)_{i} .
\end{gathered}
$$

Thus, the total effect of all these shock splittings on the functional $Q$ is bounded by

$$
\sum\left|\Delta Q_{s}^{i}\right| \leq O(1)(L(0))^{2} \leq O(1)\left(\text { Tot. Var. } u_{0}\right)^{2} .
$$

Note that the above estimate is independent of how $\left\lfloor\alpha_{1}+\cdots+\alpha_{m}\right\rfloor$ splits and how large $m$ is.

As for the case when there are other wave interactions of i-waves, from 2.14, the difference of the total strength of $\gamma$ is bounded by the decrease of $F_{o}$. Therefore, the bound 2.56 also holds.

Subcase (III.2) Now we consider the case when a shock splits into several parts but then they merge into one shock wave after some wave interactions. This procedure introduces some oscillation terms in the interaction potential. A typical case is the following series of interactions or shock splittings between i-waves (see Fig 7):

$$
\begin{aligned}
\lfloor\alpha+\beta+s+\gamma\rfloor & \rightarrow\lfloor\alpha+\beta+s\rfloor+\gamma, \\
(-\alpha)+\lfloor\alpha+\beta+s\rfloor \rightarrow \beta+s, & \rightarrow \\
\gamma+\epsilon & \rightarrow\lfloor\gamma+\epsilon\rfloor, \\
s+\lfloor\gamma+\epsilon\rfloor & \rightarrow\lfloor s+\gamma+\epsilon\rfloor, \\
\beta+\lfloor s+\gamma+\epsilon\rfloor & \rightarrow\lfloor\beta+s+\gamma+\epsilon\rfloor .
\end{aligned}
$$

Here $S_{1}, S_{2}$ are the points where conv $\tilde{f}_{i}$ and $\tilde{f}_{i}$ have contact.

And similar to the above cases, suppose first that there are no other wave interactions in the meanwhile and $\delta$ is an i-wave on the right. To illustrate the idea, in the following, we assume that $\delta$ is a shock $(\delta \geq 0)$.

By definition, the difference of $Q_{s}^{i}$ through this sequence of interactions is

$$
\begin{aligned}
& \frac{1}{2} \Delta Q_{s}^{i}=Q_{s}^{i}(\gamma, \delta)+Q_{s}^{i}(\lfloor\alpha+\beta+s\rfloor, \delta)-Q_{s}^{i}(\lfloor\alpha+\beta+s+\gamma\rfloor, \delta) \\
& +Q_{s}^{i}(s, \delta)+Q_{s}^{i}(\beta, \delta)-Q_{s}^{i}(\lfloor\alpha+\beta+s\rfloor, \delta)-Q_{s}^{i}((-\alpha), \delta) \\
& +Q_{s}^{i}(\lfloor s+\gamma+\epsilon\rfloor, \delta)-Q_{s}^{i}(\epsilon, \delta)-Q_{s}^{i}(\gamma, \delta)-Q_{s}^{i}(s, \delta)-Q_{s}^{i}(\gamma, \epsilon)-Q_{s}^{i}(s,\lfloor\gamma+\epsilon\rfloor)
\end{aligned}
$$




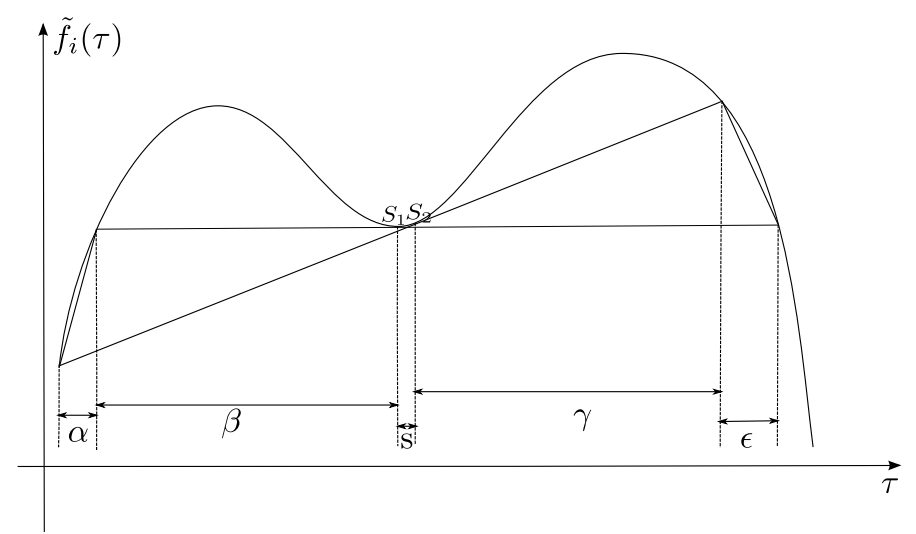

Figure 7: Wave interaction and shock splitting

$$
\begin{aligned}
& +Q_{s}^{i}(\lfloor\beta+s+\gamma+\epsilon\rfloor, \delta)-Q_{s}^{i}(\lfloor s+\gamma+\epsilon\rfloor, \delta)-Q_{s}^{i}(\beta, \delta) \\
= & Q_{s}^{i}(\lfloor\beta+s+\gamma+\epsilon\rfloor, \delta)-Q_{s}^{i}(\lfloor\alpha+\beta+s+\gamma\rfloor, \delta)-Q_{s}^{i}(\epsilon, \delta) \\
& -Q_{s}^{i}(\gamma, \epsilon)-Q_{s}^{i}(s,\lfloor\gamma+\epsilon\rfloor) \\
= & \left\{Q_{s}^{i}(\lfloor\beta+s+\gamma+\epsilon\rfloor, \delta)-\frac{Q_{o s}^{i}(\beta, \delta)+Q_{o s}^{i}(s, \delta)+Q_{o s}^{i}(\gamma, \delta)}{t . v \cdot(\beta, \delta)_{i}}-Q_{s}^{i}(\epsilon, \delta)\right\} \\
+ & \left\{\frac{Q_{o s}^{i}(\beta, \delta)+Q_{o s}^{i}(s, \delta)+Q_{o s}^{i}(\gamma, \delta)}{t . v \cdot(\beta, \delta)_{i}}-Q_{s}^{i}(\lfloor\alpha+\beta+s+\gamma\rfloor, \delta)\right\} \\
= & I+I I .
\end{aligned}
$$

Since t.v. $(\epsilon, \delta)_{i} \leq t . v \cdot(\beta, \delta)_{i}$, we have

$$
I \leq 0 .
$$

While $I I \leq O(1)|\delta||\alpha|$, where $\alpha$ is the cancellation.

Thus, modulo the cancellation, $\Delta Q_{s}^{i} \leq 0$. In fact, this reflects the fact that the interaction of same family waves in same direction always simplify the wave pattern. So it is not a coincidence.

The above computation can also be viewed from another aspect which gives a better understanding of the oscillation terms involved. That is, if we consider the interaction 2.57), 2.58, 2.59, 2.60 and 2.61 one by one and take the terms $I_{2}$ in subcase (I.1) 2.37 into account. Indeed, from the analysis in 
subcase (I), the difference of $Q_{s}^{i}$ after the interactions 2.59 and 2.60 is

$$
\begin{aligned}
\frac{1}{2} \Delta Q_{s}^{i} \leq & Q_{o s}^{i}(\epsilon, \delta)\left[\frac{1}{t \cdot v \cdot(\lfloor\gamma+\epsilon\rfloor, \delta)_{i}}-\frac{1}{t \cdot v \cdot(\epsilon, \delta)_{i}}\right] \\
& +Q_{o s}^{i}(\lfloor\gamma+\epsilon\rfloor, \delta)\left[\frac{1}{t \cdot v \cdot(\lfloor s+\gamma+\epsilon\rfloor, \delta)_{i}}-\frac{1}{t \cdot v \cdot(\lfloor\gamma+\epsilon\rfloor, \delta)_{i}}\right] \\
& -Q_{s}^{i}(\gamma, \epsilon)-Q_{s}^{i}(s,\lfloor\gamma+\epsilon\rfloor),
\end{aligned}
$$

where the first and second terms come from the term $I_{2}$ in subcase (I.1). They can be written in the following forms respectively:

$$
\begin{gathered}
Q_{o s}^{i}(\epsilon, \delta)\left[\frac{1}{t \cdot v \cdot(\lfloor\gamma+\epsilon\rfloor, \delta)_{i}}-\frac{1}{t \cdot v \cdot(\epsilon, \delta)_{i}}\right] \\
=Q_{s}^{i}(\lfloor\gamma+\epsilon\rfloor, \delta)-Q_{s}^{i}(\epsilon, \delta)-\frac{Q_{o s}^{i}(\lfloor\gamma+\epsilon\rfloor, \delta)-Q_{o s}^{i}(\epsilon, \delta)}{t \cdot v \cdot(\lfloor\gamma+\epsilon\rfloor, \delta)_{i}} \\
=Q_{s}^{i}(\lfloor\gamma+\epsilon\rfloor, \delta)-Q_{s}^{i}(\epsilon, \delta)-Q_{s}^{i}(\gamma, \delta)+O(1) \delta Q_{s}^{i}(\gamma, \epsilon), \\
Q_{o s}^{i}(\lfloor\gamma+\epsilon\rfloor, \delta)\left[\frac{1}{t \cdot v \cdot(\lfloor s+\gamma+\epsilon\rfloor, \delta)_{i}}-\frac{1}{t \cdot v \cdot(\lfloor\gamma+\epsilon\rfloor, \delta)_{i}}\right] \\
=Q_{s}^{i}(\lfloor s+\gamma+\epsilon\rfloor, \delta)-Q_{s}^{i}(\lfloor\gamma+\epsilon\rfloor, \delta)-\frac{Q_{o s}^{i}(\lfloor s+\gamma+\epsilon\rfloor, \delta)-Q_{o s}^{i}(\lfloor\gamma+\epsilon\rfloor, \delta)}{t \cdot v \cdot(\lfloor s+\gamma+\epsilon\rfloor, \delta)_{i}} \\
=Q_{s}^{i}(\lfloor s+\gamma+\epsilon\rfloor, \delta)-Q_{s}^{i}(\lfloor\gamma+\epsilon\rfloor, \delta)-Q_{s}^{i}(s, \delta)+O(1) \delta Q_{s}^{i}(s,\lfloor\gamma+\epsilon\rfloor),
\end{gathered}
$$

where we have used Corollary 2.1 as in subcase (I.1). This is essentially the same as our previous computation.

Combine subcase (III.1) and (III.2), we can see that the total contribution of shock splittings can be bounded by $(2.56)$ in any time interval $[0, T]$.

The proof of the interaction estimate in general case is the combination of the above typical cases.

From the proof of Theorem 1.1. we can divide the functional $Q_{s}$ into two functionals which are useful in the wave tracing argument.

Corollary 2.2. In any time interval $[0, T]$, denote the time when the interaction of $i$-th family waves or the splitting of $i$-th family shocks happens by $t_{k}^{i}, k \in \mathbb{N}, i=$ $1, \cdots, n$. Define a functional $S(t)$ with $S(0)=0 . S$ is unchanged except at $t_{k}^{i}$. At $t_{k}^{i}$, we define the quantity $S\left(t_{k}^{i}+\right)=S\left(t_{k}^{i}-\right)+\Delta S\left(t_{k}^{i}\right)$ by distinguishing two cases: 
(i) In the case of wave interaction of i-family waves as in Figure 4, we define

$$
\begin{aligned}
& \Delta S\left(t_{k}^{i}\right) \\
= & \sum_{l} 2 \int_{\substack{\left[0, \varepsilon_{l}\right] \\
\text { or }\left[\varepsilon_{l}, 0\right]}} \int_{\substack{[0, \alpha] \\
\text { or }[\alpha, 0]}}\left\{\frac{\left|\sigma_{\varepsilon_{l}}\left(\tau^{\prime}\right)-\sigma_{\alpha}(\tau)\right|}{t . v \cdot\left(T_{\varepsilon_{l}}^{i}\left[u_{\varepsilon_{l}}\right]\left(\tau^{\prime}\right), T_{\beta}^{i}\left[u_{\beta}\right](\beta)\right)_{i}}-\frac{\left|\sigma_{\varepsilon_{l}}\left(\tau^{\prime}\right)-\sigma_{\alpha}(\tau)\right|}{t . v \cdot\left(T_{\varepsilon_{l}}^{i}\left[u_{\varepsilon_{l}}\right]\left(\tau^{\prime}\right), T_{\alpha}^{i}\left[u_{\alpha}\right](\tau)\right)_{i}}\right\} \mathrm{d} \tau \mathrm{d} \tau^{\prime} .
\end{aligned}
$$

Similarly, if there are i-family waves $\varepsilon_{k}$ on the right of $\alpha$ and $\beta$, we define

$$
\begin{aligned}
& \Delta S\left(t_{k}^{i}\right) \\
= & \sum_{k} 2 \int_{\substack{\left[0, \varepsilon_{k}\right] \\
\text { or }\left[\varepsilon_{k}, 0\right]}} \int_{\substack{[0, \beta] \\
\text { or }[\beta, 0]}}\left\{\frac{\left|\sigma_{\beta}(\tau)-\sigma_{\varepsilon_{k}}\left(\tau^{\prime}\right)\right|}{t . v \cdot\left(T_{\beta}^{i}\left[u_{\alpha}\right](\alpha), T_{\varepsilon_{k}}^{i}\left[u_{\varepsilon_{k}}\right]\left(\tau^{\prime}\right)\right)_{i}}-\frac{\left|\sigma_{\beta}(\tau)-\sigma_{\varepsilon_{k}}\left(\tau^{\prime}\right)\right|}{t \cdot v \cdot\left(T_{\beta}^{i}\left[u_{\beta}\right](\tau), T_{\varepsilon_{k}}^{i}\left[u_{\varepsilon_{k}}\right]\left(\tau^{\prime}\right)\right)_{i}}\right\} \mathrm{d} \tau \mathrm{d} \tau^{\prime} .
\end{aligned}
$$

And in general, when there are ith-family waves on both left and right of $\alpha$ and $\beta$, the $\Delta S\left(t_{k}^{i}\right)$ is the summation of the above two terms.

(ii) In the case of shock splitting of i-family as in Figure 1: $\alpha \rightarrow \alpha_{1}+\alpha_{2}$, we define

$$
\begin{aligned}
& \Delta S\left(t_{k}^{i}\right) \\
= & 2 \sum_{\gamma} \int_{\substack{[0, \gamma] \\
\text { or }[\gamma, 0]}}\left(\frac{1}{t . v \cdot\left(\alpha_{2}, T_{\gamma}^{i}\left[u_{\gamma}\right](s)\right)_{i}}-\frac{1}{t . v \cdot\left(\left\lfloor\alpha_{1}+\alpha_{2}\right\rfloor, T_{\gamma}^{i}\left[u_{\gamma}\right](s)\right)_{i}}\right)\left|\alpha_{2}\right|\left|\sigma_{\alpha}-\sigma_{\gamma}(s)\right| \mathrm{d} s .
\end{aligned}
$$

Then we can define a functional $S(t)$ for all time $t$. With $S$, we can define another non-decreasing functional $G$.

In any time interval $[0, T]$, denote by $\tilde{t}_{k}, k \in \mathbb{N}$ the time when the interaction of different family waves happens. $G$ is unchanged except at $t_{k} \in\left\{t_{k}^{i} ; k \in \mathbb{N}, i=\right.$ $1, \cdots, n\} \cup\left\{\tilde{t}_{k} ; k \in \mathbb{N}\right\}$ and

$$
\begin{gathered}
G(0)=F(0), \quad G\left(t_{k}+\right)=G\left(t_{k}-\right)+\Delta G\left(t_{k}\right), \\
\Delta G\left(t_{k}\right) \equiv \Delta S\left(t_{k}\right)-\Delta F\left(t_{k}\right),
\end{gathered}
$$

where $\Delta F\left(t_{k}\right)=F\left(t_{k}+\right)-F\left(t_{k}-\right)$ as usual. At any $t_{k}$ when the waves $\alpha$ and $\beta$ interact, we have

$$
\Delta G\left(t_{k}\right) \geq c(Q(\alpha, \beta)+C(\alpha, \beta)) \geq 0,
$$


where $c>0$ is a constant depending only on the system. And for any $T>0$,

$$
\begin{gathered}
S(T) \leq O(1)\left(\text { Tot. Var. } u_{0}\right)^{2}, \\
0 \leq G(T) \leq O(1)\left(\text { Tot. Var. } u_{0}\right)^{2},
\end{gathered}
$$

where $u_{0}$ is the initial data and the $0(1)$ constant is independent of $T$.

Furthermore, in the case I.1 as in Figure 4, we have

$$
\int_{0}^{\alpha+\beta}\left|\sigma_{\delta_{i}}(s)-\left(\sigma_{\alpha} \cup \sigma_{\beta}\right)(s)\right| \mathrm{d} s \leq O(1) \Delta G\left(t_{k}^{i}\right) .
$$

Proof. In the proof, without loss of generality, we assume that there is only one wave interaction at a given interaction time. On the other hand, if there are more than one wave interactions happen at one time, the change of the functional is just the sum over all the changes through all the interactions at that time.

By the estimate of I 2.37) in Theorem 1.1 case (I), we see that in case (I)

$$
\Delta F\left(t_{k}^{i}\right) \leq\left\{-c\left(Q_{s}^{i}(\alpha, \beta)+C(\alpha, \beta)\right)\right\}+\Delta S\left(t_{k}^{i}\right),
$$

when Tot. Var. $(u)$ is sufficiently small. Here $\Delta S\left(t_{k}^{i}\right)$ comes from the estimate of $I_{2}$ in the proof of Theorem 1.1 case (I.1). As for Theorem 1.1 case (III), it is easy to see that

$$
\Delta F\left(t_{k}^{i}\right)=\Delta S\left(t_{k}^{i}\right)
$$

In both cases, we have

$$
\Delta G\left(t_{k}^{i}\right)=\Delta S\left(t_{k}^{i}\right)-\Delta F\left(t_{k}^{i}\right) \geq c\left(Q_{s}^{i}(\alpha, \beta)+C(\alpha, \beta)\right) \geq 0 .
$$

On the other hand, at the time $\tilde{t}_{k}$ when different family waves interacts as in Figure 5, we have

$$
\Delta G\left(t_{k}\right)=-\Delta F\left(t_{k}\right) \geq c Q_{d}(\alpha, \beta) \geq 0 .
$$

Therefore, we define a non-decreasing functional $G$ at any time when the waves interact or shocks split and 2.72 holds. And 2.73 can be justified by applying the argument used in 2.64. Then 2.73) and 2.74 are the direct consequences of the definition and the estimates 1.8 and 2.56 .

The proof of 2.75 is similar to the proof of Theorem 3.3 in 14. By definition, in case (I.1), it suffices to consider the case that $\delta_{i}$ is a single shock. In this case, by 2.15), 


$$
\sigma_{\delta_{i}}=\frac{\eta(\alpha)+\eta(\beta)}{\alpha+\beta}+O(1) \frac{Q_{o s}^{i}(\alpha, \beta)}{\alpha+\beta} .
$$

And as discussed in Corollary 2.1, the monotonicity property of $\sigma_{\alpha}$ and $\sigma_{\beta}$ implies that

$$
\begin{aligned}
& \int_{0}^{\alpha+\beta}\left|\sigma_{\delta_{i}}(s)-\left(\sigma_{\alpha} \cup \sigma_{\beta}\right)(s)\right| \mathrm{d} s \\
= & \int_{0}^{\alpha}\left(\sigma_{\alpha}(s)-\sigma_{\delta_{i}}(s)\right) \mathrm{d} s+\int_{0}^{\beta}\left(\sigma_{\delta_{i}}(\alpha+s)-\sigma_{\beta}(s)\right) \mathrm{d} s \\
= & 2 \frac{\beta \eta(\alpha)-\alpha \eta(\beta)}{\alpha+\beta}+O(1) \frac{Q_{o s}^{i}(\alpha, \beta)}{\alpha+\beta} \\
\leq & O(1) Q_{s}^{i}(\alpha, \beta) \leq O(1) \Delta G\left(t_{k}^{i}\right) .
\end{aligned}
$$

And this completes the proof of the corollary.

\section{Application}

As an application of the Glimm functional $(2.22)$, in this section, we study the convergence rate of Glimm scheme.

To state the theorem, the following equi-distributed random sequence is used, cf. [8].

Lemma 3.1. Let

$$
D_{m, n}=\sup _{\lambda \in[0,1]}\left|\lambda-\frac{1}{n-m} \sum_{m \leq l<n} \chi_{[0, \lambda]}\left(\theta_{l}\right)\right|,
$$

then there exists a sequence $\left\{\theta_{l}\right\}_{l \geq 0} \subset[0,1]$ such that

$$
D_{m, n} \leq O(1) \frac{1+\ln (n-m)}{n-m} \quad \forall n>m \geq 1 .
$$

Similar to 22, all the waves in the solution are partitioned into small subwaves as follows.

Definition 3.1. Let $u_{r}=T_{s}^{i}\left[u_{l}\right](s) .\left(u_{l}, u_{r}\right)$ is an i-wave. A set of vectors $\left\{v_{0}, v_{1}, \cdots, v_{p}\right\}$ is a partition of $\left(u_{l}, u_{r}\right)$ if

(i) $v_{0}=u_{l}, v_{p}=u_{r}$, 
(ii) there exists a set of scalars $\left\{\tau^{0}, \tau^{1}, \tau^{2}, \cdots, \tau^{p}\right\}$ such that

$$
\begin{gathered}
0=\tau^{0} \leq \tau^{1} \leq \tau^{2} \leq \cdots \leq \tau^{p}=s, \text { for } s>0 ; \\
0=\tau^{0} \geq \tau^{1} \geq \tau^{2} \geq \cdots \geq \tau^{p}=s, \text { for } s<0 ; \\
v_{h}=T_{s}^{i}\left[u_{l}\right]\left(\tau^{h}\right), \text { for } h=1, \cdots, p .
\end{gathered}
$$

Then set

(1) $y_{h}=v_{h}-v_{h-1}, s_{h}=\tau^{h}-\tau^{h-1}$, for $h=1, \cdots, p$,

(2) $\lambda_{i, h} \equiv \frac{1}{s_{h}} \int_{\tau^{h-1}}^{\tau^{h}} \sigma_{i}\left(\xi ; s, u_{l}\right) \mathrm{d} \xi$,

where $s_{h}, \lambda_{i, h}$ are the strength and speed of the subwave $y_{h}$.

Then as a consequence of Theorem 1.1, we have the following theorem.

Theorem 3.1. The waves in an approximate solution in a given a time zone $\Lambda=\{(x, t):-\infty<x<\infty, M s \leq t<(M+N) s\}$, for any given integers $M$ and $N$, can be partitioned into subwaves of categories I, II or III with the following properties:

(i). The subwaves in I are surviving. Given a subwave $\alpha(t), M s \leq t<$ $(M+N) s$, in $I$, write $\alpha \equiv \alpha(M s)$ and denote by $|\alpha(t)|$ its strength at time $t$, by $[\sigma(\alpha)]$ the variation of its speed and by $[\alpha]$ the variation of the jump of the states across it over the time interval $M s \leq t<(M+N) s$. Then

$$
\sum_{\alpha \in I}([\alpha]+|\alpha(M s)|[\sigma(\alpha)])=O(1)(D(\Lambda)+s),
$$

where $D(\Lambda)=G((M+N) s-)-G(M s+)$.

(ii). A subwave $\alpha(t)$ in II has non-zero initial strength $|\alpha(M s)|>0$, but is cancelled in the zone $\Lambda,|\alpha((M+N) s)|=0$. Moreover, the total strength and variation of the wave speed satisfy

$$
\begin{gathered}
\sum_{\alpha \in I I}([\alpha]+|\alpha(t)|)=O(1)(D(\Lambda)+s), M s \leq t<(M+N) s \\
\sum_{\alpha \in I I}([\alpha]+|\alpha(M s)|[\sigma(\alpha)]) \leq O(1)(D(\Lambda)+s) .
\end{gathered}
$$

(iii). A subwave in III has zero initial strength $|\alpha(M s)|=0$, and is created in the zone $\Lambda,|\alpha((M+N) s)| \neq 0$. Moreover, the total strength and variation of the wave speed satisfy

$$
\sum_{\alpha \in I I I}([\alpha]+|\alpha(t)|)=O(1)(D(\Lambda)+s), M s \leq t<(M+N) s,
$$




$$
\sum_{\alpha \in I I I}([\alpha]+|\alpha((M+N) s)|[\sigma(\alpha)]) \leq O(1)(D(\Lambda)+s) .
$$

By using Corollary 2.2, the proof of this theorem is similar to the one in 14 . So we omit the proof for brevity. By the argument used in [8], we can get the convergence rate of the deterministic version of Glimm scheme as stated in the following theorem. The only difference is that now we use $D(\Lambda)=\Delta G$ instead of $|\Delta F|$. We omit its proof for brevity.

Theorem 3.2. Let $\left\{\theta_{m}\right\}_{m=1}^{\infty}$ be a sequence of numbers in $[0,1]$ satisfying $(3.2)$. Given any initial condition $\bar{u}$ with small total variation, let $u(\cdot, t)=S_{t} \bar{u}$ be the unique solution of (1.1), and let $u^{s}$ be the corresponding Glimm approximate solution with grid size s in the time direction, generated by the sampling sequence $\left\{\theta_{m}\right\}_{m=1}^{\infty}$. Then for every $T \geq 0$,

$$
\lim _{s \rightarrow 0} \frac{\left\|u^{s}(\cdot, T)-u(\cdot, T)\right\|_{L^{1}}}{s^{\frac{1}{2}}|\ln s|}=0 .
$$

The limit is uniform with respect to $\bar{u}$, as long as T.V.ü remains uniformly small.

Acknowledgments: The research was supported by the the Strategic Research Grant of City University of Hong Kong No. 7002447.

\section{References}

[1] F. Ancona AND A. MARson, Sharp convergence rate of the glimm scheme for general nonlinear hyperbolic systems. Available at http://www.math.ntnu.no/conservation/2009/012.html, 2009.

[2] S. Bianchini, Interaction estimates and Glimm functional for general hyperbolic systems, Discrete Contin. Dyn. Syst., 9 (2003), pp. 133-166.

[3] - On the Riemann problem for non-conservative hyperbolic systems, Arch. Ration. Mech. Anal., 166 (2003), pp. 1-26.

[4] S. Bianchini And A. Bressan, Vanishing viscosity solutions of nonlinear hyperbolic systems, Ann. of Math. (2), 161 (2005), pp. 223-342.

[5] A. Bressan, Hyperbolic systems of conservation laws, vol. 20 of Oxford Lecture Series in Mathematics and its Applications, Oxford University Press, Oxford, 2000. 
[6] A. Bressan, G. Crasta, And B. Piccoli, Well-posedness of the Cauchy problem for $n \times n$ systems of conservation laws, Mem. Amer. Math. Soc., 146 (2000), pp. viii+134.

[7] A. Bressan, T.-P. Liu, And T. YAng, $L^{1}$ stability estimates for $n \times n$ conservation laws, Arch. Ration. Mech. Anal., 149 (1999), pp. 1-22.

[8] A. Bressan and A. Marson, Error bounds for a deterministic version of the Glimm scheme, Arch. Rational Mech. Anal., 142 (1998), pp. 155-176.

[9] R. Courant and K. O. Friedrichs, Supersonic Flow and Shock Waves, Interscience Publishers, Inc., New York, N. Y., 1948.

[10] C. M. Dafermos, Hyperbolic conservation laws in continuum physics, vol. 325 of Grundlehren der Mathematischen Wissenschaften [Fundamental Principles of Mathematical Sciences], Springer-Verlag, Berlin, 2000.

[11] R. J. DiPerna, Convergence of approximate solutions to conservation laws, Arch. Rational Mech. Anal., 82 (1983), pp. 27-70.

[12] J. GLImm, Solutions in the large for nonlinear hyperbolic systems of equations, Comm. Pure Appl. Math., 18 (1965), pp. 697-715.

[13] J. Glimm And P. D. Lax, Decay of solutions of systems of nonlinear hyperbolic conservation laws, Memoirs of the American Mathematical Society, No. 101, American Mathematical Society, Providence, R.I., 1970.

[14] J. Hua, Z. JiAng, And T. YAng, A new Glimm functional and convergence rate of Glimm scheme for general systems of hyperbolic conservation laws. To appear in Arch. Ration. Mech. Anal, 2009.

[15] J. Hua And T. YAng, An improved convergence rate of Glimm scheme for general systems of hyperbolic conservation laws, J. Differential Equations, 231 (2006), pp. 92-107.

[16] T. IGUChi And P. G. LeFloch, Existence theory for hyperbolic systems of conservation laws with general flux-functions, Arch. Ration. Mech. Anal., 168 (2003), pp. 165-244.

[17] S. N. KružKov, First order quasilinear equations with several independent variables., Mat. Sb. (N.S.), 81 (123) (1970), pp. 228-255. 
[18] P. D. Lax, Hyperbolic systems of conservation laws. II, Comm. Pure Appl. Math., 10 (1957), pp. 537-566.

[19] T. P. Liu, The deterministic version of the Glimm scheme, Comm. Math. Phys., 57 (1977), pp. 135-148.

[20] — Admissible solutions of hyperbolic conservation laws, Mem. Amer. Math. Soc., 30 (1981), pp. iv+78.

[21] T.-P. LiU And T. YAnG, Well-posedness theory for hyperbolic conservation laws, Comm. Pure Appl. Math., 52 (1999), pp. 1553-1586.

[22] — Weak solutions of general systems of hyperbolic conservation laws, Comm. Math. Phys., 230 (2002), pp. 289-327.

[23] O. A. OLEINIK, Uniqueness and stability of the generalized solution of the Cauchy problem for a quasi-linear equation, Uspehi Mat. Nauk, 14 (1959), pp. $165-170$.

[24] J. Smoller, Shock waves and reaction-diffusion equations, vol. 258 of Grundlehren der Mathematischen Wissenschaften [Fundamental Principles of Mathematical Science], Springer-Verlag, New York, 1983.

[25] T. YAnG, Convergence rate of Glimm scheme for general systems of hyperbolic conservation laws, Taiwanese J. Math., 7 (2003), pp. 195-205. 